\title{
Road-side units operators in competition: A game-theoretical approach
}

\author{
Q1 Vladimir Fux ${ }^{a}$, Patrick Maillé ${ }^{a}$, Matteo Cesana ${ }^{\text {,** }}$ \\ a Institut Mines-Telecom/Telecom Bretagne, Rennes, France \\ ${ }^{\mathrm{b}}$ Dip. di Elettronica, Informazione e Bioingegneria, Politecnico di Milano, Italy
}

\section{A R T I C L E I N F O}

\section{Article history:}

Received 19 December 2014

Revised 15 June 2015

Accepted 16 June 2015

Available online $\mathrm{xxx}$

\section{Keywords:}

Vehicular networks

Game theory

Pricing

Competition

\begin{abstract}
A B S T R A C T
We study the interactions among Internet providers in vehicular networks which offer access to commuters via road side units (RSUs). Namely, we propose a game-theoretical framework to model the competition on prices between vehicular Internet providers to capture the largest amount of users, thus selfishly maximizing the revenues. The equilibria of the aforementioned game are characterized under different mobile traffic conditions, RSU capabilities and users requirements and expectations. In particular, we also consider in the analysis the case where mobile users modify the price they accept to pay for the access as the likeliness of finding an access solution decreases.

Our game-theoretical analysis gives insights on the outcomes of the competition between vehicular Internet providers, further highlighting some counter-intuitive behaviors; as an example, comparing with the case when users have constant price valuation over time, having users inclined to increasing their "acceptable" price may force vehicle Internet providers to charge lower prices due to competition.
\end{abstract}

(c) 2015 Elsevier B.V. All rights reserved.

\section{Introduction}

Vehicular Ad-hoc NETworks (VANETs) recently attracted much interest from the research community as a core networking component to build up intelligent transportation systems (ITS) to improve road safety, optimize the humans and goods mobility, and disseminate real-time context information on traffic loads, congestion and hazardous situations. The applications enabled by VANETs are not only limited to safety-oriented ones, but also extend to leisure applications related to Internet access and entertainment along the road. A comprehensive classification of VANETs applications can be found in [12].

\footnotetext{
* Corresponding author. Tel.: +39 223993695; fax: +39 223993413

E-mail address: cesana@elet.polimi.it, macesana@gmail.com, matteo.cesana@polimi.it (M. Cesana).
}

The design of VANET architectures to support leisure applications has attracted the attention of recent work and researchers; as an example, the Drive-thru Internet [22] project targets the provision of affordable Internet connections to vehicular users through road side Wireless LAN infrastructure. The scope of the research covers network access, roaming, handover, authentication, etc., and the achieved results show that despite a number of technical challenges to be addressed, providing Internet for highly mobile vehicular users is possible [21-23,25]. The CABERNET [7] and Infostations [28] projects propose architectures similar to Drive-Thru Internet. Motivated by these works, we expect that the provision of Internet connectivity via road side infrastructure will be a flourishing market in the next future attracting Internet providers which may possibly compete among themselves. This competition may have a valuable impact on customers welfare, as well as influence the quality and cost of all aforementioned features about road safety. 
The scientific literature already counts a number of studies of competition between classical Internet access providers (see, e.g., [1,15] or [16, Chapter 5]). In many cases, the interactions among users (through congestion) are also considered, and taken into account by access providers $[9,10]$. However, to the best of our knowledge the case of provider competition in vehicular networks has not been deeply investigated, although it has some important specificities; indeed customers are mobile and move in a limited speed range and, more importantly, in constrained directions. In this work we want to fill this gap by providing a study of duopoly competition, between providers owning one road side unit (RSU) each, along a stretch of road. These road side units are able (besides all other features) to provide Internet access to mobile users, whose cars are equipped with a device called on-board unit (OBU). We study how providers strategically set their price for providing Internet connectivity in response to the competitor's pricing strategy with the selfish objective of revenue maximization; vehicular users may decide to get Internet connectivity from one operator or the other depending on the corresponding price and the current network conditions. This manuscript builds on our preliminary work in [11], further extending the network scenario by considering that users can change their acceptance/refusal strategy (or equivalently, their price preferences) while they travel along the stretch of road. We investigate how this variation influences the pricing strategies of providers. Such a question is linked to the specificities of vehicular networks, and to the best of our knowledge has not been studied in the scientific literature. Among the unexpected results, we observed that users increasing their price acceptance threshold between the two RSUs, if anticipated by providers, strongly impacts the competition among them and can lead to lower prices and lower provider revenues (with respect to the case when users have fixed price acceptance thresholds).

The manuscript is organized as follows: Section 2 gives an overview of the related work further commenting on the main novelties and contributions of the present work; Section 3 introduces the reference scenario and the related modeling assumptions; in Section 4, we analyze the case where the pricing policy of one vehicular Internet provider is fixed and the competitor best-responds to it. Section 5 analyzes the non-cooperative game between vehicular Internet providers, focusing on the consequences in terms of provider revenues and user welfare. Further comments on the modeling assumptions and concluding remarks are reported in Section 6.

\section{Related work}

Though vehicular networks are far from being widely deployed, the research community already started to extensively study different problems and challenges likely to arise in the future. Many articles are devoted to the definition/adaptation of communication protocols for the vehicular context (like in [3,14,33-35]), studying the suitability of already existing technologies and proposing new approaches. The main challenge here is to develop a reliable protocol for V2V communications.
The suitability of WLAN hotspots for providing Internet access in vehicular scenario is studied in $[7,22,28]$. In [22], mobile users exploit temporary WLAN connections during their road trip to download/upload contents form/to the Internet; the main challenge addressed in this work is to maintain a seamless connectivity even if the physical connection with a road side access point may get lost temporarily. Along the same lines, automatic access point association/deassociation procedures are studied in [24,26] in the very same vehicular network architecture. Besides a purely theoretical studies, special equipments for highly mobile scenarios are in development, among which a router with 3G and WLAN interfaces is designed to ensure seamless handovers, proposed by NEC Corporation in 2005. In [25], the authors discuss the requirements for such a router and test their own prototype of modular access gateway.

Another research area related to this work deals with the optimal design of vehicular networks, where the problem mainly scales down to efficiently deploying RSU to maximize the "quality" perceived by the mobile user in terms of download/upload throughput, and/or latency to retrieve contents form the Internet through the deployed RSUs. Trullols et al. [30] consider different formulations for the deployment problem and introduce heuristics based on local-search and greedy approaches to get suboptimal solutions. A solution based on genetic algorithms is studied by Cavalcante et al. [4]. Yan et al. [32] study the optimal RSU deployment problem, where candidate places for RSU location are crossroads. A comprehensive description of the general problem of optimal RSU deployment by a single entity can be found in [2] and [36]. A different scenario, where several providers deploy their RSUs in a competitive manner is studied in [8], and the same problem but for general wireless networks is considered in [1].

Researchers often use game theory to study competition between providers. In [19] the authors survey various gametheoretic models for evaluating the competition between agents in vehicular networks. The mobile users competition is studied in [20], where users share the same RSU. In [18] a hierarchical game is proposed to analyze the competition between OBUs and RSUs. Differently, in [27] a coalition formation game among RSU is analyzed, with the aim of better exploiting V2V communications for data dissemination. More generally, good surveys on game theory applications in wireless networks are [5] and [29].

In this paper, unlike in the previously described references we ignore V2V communications and focus only on users which aim to establish Internet connection. In that context, we consider price competition between Internet access providers in the case of vehicular networks, which is, to the best of our knowledge, a novel issue. The scientific literature contains several analyses of provider competition in general wireless networks (e.g., [6,17,31]), but, even if V2I networks bear some similarities with generic wireless access networks, they have specific features which make the pricing problem worth analyzing. Indeed, in generic wireless access networks, the network operator competition is generally over the "common" users, that is, those users which fall in the coverage area of the competing network providers. In other words, competition between providers arise only if the coverage areas of the networks (partially) overlap as in [17]. Users 
themselves tend to select an access point which maximizes some quality measure as in [9]. On the other hand, in V2I networks competition may arise due to vehicles mobility even if the coverage areas of competing RSUs do not overlap, since if an RSU does not serve a moving vehicle in its own coverage range, the very same user can be served later by competing operators; in this case users do not really make a network selection decision, rather they answer the binary question of whether or not to connect to the currently observed network.

In contrast to [11], where we analyze competition among Internet access providers, in the current study we also focus on customers and their welfare. We assume that mobile users may deviate from their original pricing preferences after receiving additional information about the connection cost. More specifically, we consider that the users are somehow risk-averse and can modify their connection budget after passing an access point without being served. This modification, if it is a common feature/strategy of users population, may lead to several interesting outcomes and peculiarities, such as connection prices drops and, sequentially, providers revenue losses.

\section{Reference scenario and modeling assumptions}

We consider a stretch of a highway where two Internet access providers coexist. However, our model is applicable for scenarios where the number of RSUs at each provider's disposal is arbitrary, even with non-overlapping coverage areas, with the constraint that available providers are not alternating along the road, that is, users may cross several regions covered by Provider 1, then several covered by Provider 2 (or vice-versa). This model represents the case of local access providers along a freeway for example; the case of RSUs from alternating providers is not covered here, and is left for future work.

Note that in this article we do not treat the cases when more than two Internet access providers compete. In such cases the RSU location would be of high importance, which we highlight here by briefly evoking a scenario with three providers. The provider whose RSU is located between the two others is obviously in a disadvantageous position, since he can only serve users who were unserved by competitors. For example, in the case of low user flows (no congestion), the "middle" provider only sees users with low willingnessto-pay (since they refused the offer of the first provider they met) and should therefore set relatively low prices. In the general case, this "middle" provider would absorb some of the unserved traffic of the two others, hence reducing the interactions between the extremity providers. Since those interactions are the focus of this paper, we believe the twoprovider case highlights better the specificities of vehicular networks (with users arriving from both directions and affecting the relationships among providers). Finally, the twoprovider case is sufficiently simple to allow us to reach analytical results, while considering more providers is likely to be treatable only through numerical studies.

For the sake of easing up presentation, we assume that RSUs are totally identical and have the same individual goodput (or capacity) $c$. It is worth pointing out that the modeling framework can be extended to the case where the RSUs owned by the different providers have different capacity values. The providers' RSU locations differ, and thus vehicles taking the road in one direction first enter the coverage area of Provider 1's RSU, while those traveling in the opposite direction first see Provider 2 . We denote by $\lambda_{j}, j=1,2$ the average number of commuters per time unit that first meet Provider j's RSU; they will cross the competitor's coverage area afterwards. Note that we will treat those average arrivals number as constant, i.e., we reason as if there are exactly $\lambda_{j}$ commuters per time unit seeing Provider $j$ first.

Each user wants to establish an Internet connection to download data files. The average volume of these files per user is normalized to 1 without loss of generality, and we will also treat the file volume as a constant. Hence the total demand (in term of data volume) of users seeing Provider $j$ first is also $\lambda_{j}$. We assume that the RSUs coverage area and the vehicles' speed do not constrain file transfers: if a RSU's capacity exceeds its (average) load, all requests are successfully served, otherwise some requests (taken randomly) are rejected.

Each provider $j=1,2$ set a (flat-rate) price $p_{j}$ to charge for the connection service. However not all users will accept this price. We model users price preferences by assuming that only a proportion $w(p)$ of users accept to pay a unit price $p$ for the service. If Provider $j$ charges price $p_{j}$, users who first enter Provider $j$ 's service area generate a demand (again, per time unit, and treated as static) of $w\left(p_{j}\right) \lambda_{j}$. The function $w($.$) is called willingness-to-pay function, and we assume$ it to be non-increasing: each user can be seen as having a maximum price below which he/she accepts the service, and above which he/she refuses to connect, the function $w(\cdot)$ then represents the complementary cumulative distribution function of those acceptance prices among users.

\subsection{Demand flows}

Fig. 1 summarizes the scenario in terms of demand flows. The total flow $\lambda_{j}$ from users seeing first Provider $j$ consists of:

1. users accepting the price $p_{j}$ and being served by Provider $j$;

2. users accepting the price $p_{j}$ and being rejected due to the RSU capacity limit (forming a spillover flow $\lambda_{j}^{\mathrm{sp}}$ heading to the competitor's RSU);

3. and users refusing the price $p_{j}$ (forming a flow $\lambda_{j}^{\text {ref }}$ heading to the competitor's RSU).

The two latter flows then enter the coverage area of the competing provider, where they can be served or not.

We consider here that users may change their price acceptance threshold after meeting one provider and having either refused its price or been rejected due to capacity limits. In the following, we analyze both cases in which refused/rejected users increase and decrease their willingness to pay as they go by. It is worth noting that these behaviors are well representative of realistic situations:

- willingness-to-pay increases, if the user's request was rejected due to congestion, this signal of resource scarcity may increase the user's willingness-to-pay; alternatively, users may know that there are several RSUs on the highway they are using, and hence may "take a bet" for the first RSU they meet, by being more demanding than they 


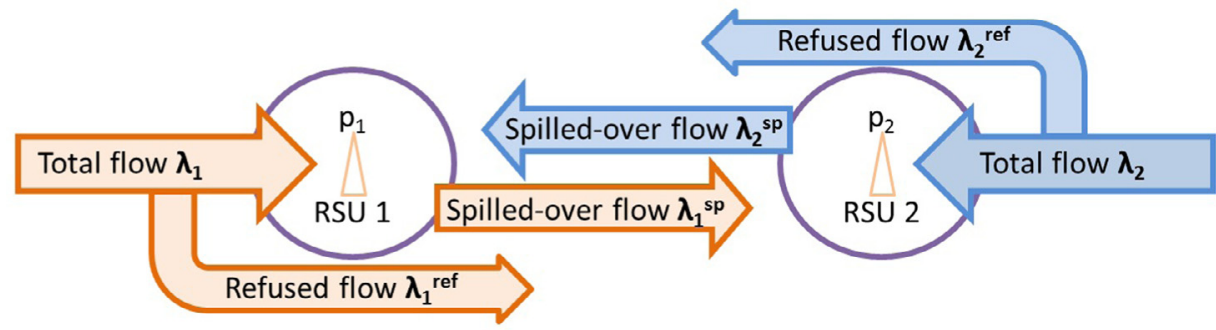

Fig. 1. Flows involved in the model: among the total potential demand $\lambda_{j}$ seeing Provider $j$ first, we distinguish $\lambda_{j}^{\text {sp }}$ (demand from users agreeing to pay $p_{j}$, but not served by this provider), $\lambda_{j}^{\text {ref }}$ (demand from users refusing to pay $p_{j}$ ). All users unserved after passing Provider $j$ increase their willingness-to-pay. We define the same flows, indexed by $k$, for users traveling in the opposite direction.
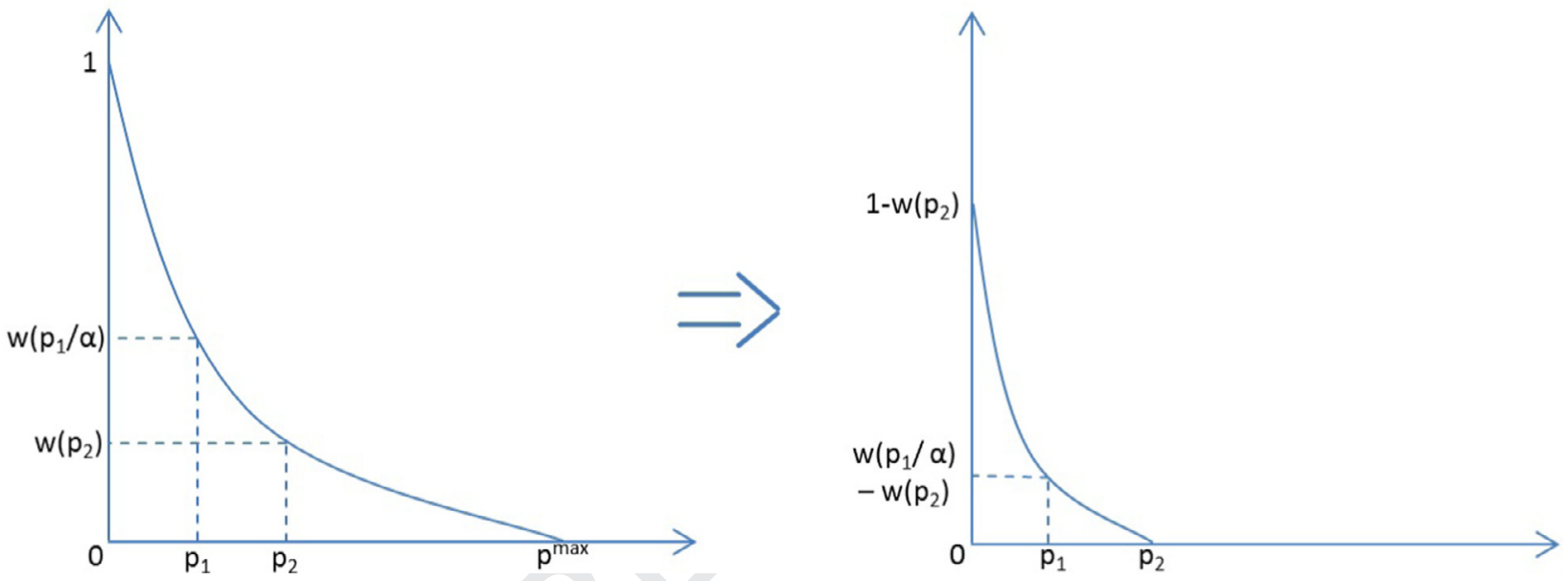

Fig. 2. How willingness to pay for users flow changes after passing e.g. RSU 2.

could really afford. The logic in this case is that probably the next RSUs are cheaper. As more RSUs are crossed, the risk raises to find no other RSU (or only more expensive ones) before some delay limit, hence a higher price acceptance threshold after passing each RSU;

- willingness-to-pay decreases, if the content the user is requesting is time-sensitive, that is, the user wants a specific content at a specific time, the additional delay on content retrieval the user experiences for being rejected/refused may lead the user to value less the content/connectivity.

This change in willingness-to-pay impacts two components of the total available demand at a provider-refused and spilled-over users from the competitor-, making them more (or less) valuable for the provider (who may extract more or less revenue from those users). Note that this can be easily extended to a scenario when each provider owns several (consecutive) RSUs; there, each user would change his willingness-to-pay when changing provider, not RSUs.

In this paper, we consider a simple multiplicative change of the acceptance threshold:

- if a user refused to pay the price of the first RSU he/she met, his price acceptance threshold is multiplied by $\alpha$;

- if a user accepted the price of an RSU but his request was rejected due to congestion, his price acceptance threshold is multiplied by $\beta$.
To simplify a bit the analysis, we assume in the following that $\alpha=\beta$, i.e., users that are not served modify their acceptance threshold price by the same factor, whether they had accepted or refused the price of the first RSU they met. Such an assumption is realistic, if the price variation is interpreted as a response to the decreasing likelihood of finding another (cheap) RSU.

It is worth pointing out that if all users simultaneously accept to pay a price $\alpha$ times larger (smaller) than before, then the proportion of users accepting to pay $p$ is changed from $w(p)$ to $w\left(\frac{p}{\alpha}\right)$. Fig. 2 shows an example of how the willingness-to-pay function changes after users have passed RSU 2, when no congestion occurs at RSU 2. Some of the users seeing Provider 2 first (a proportion $w\left(p_{2}\right)$ of them) accepted to pay the price of Provider 2 and were served, and thus do not need a connection anymore. The others increase the maximum price they can afford by $\alpha$ : the proportion of users seeing Provider 2 first and accepting to pay price $p_{1}$ is then $w\left(p_{1} / \alpha\right)-w\left(p_{2}\right)$.

We now decompose formally the components of the user flows reaching Provider $j$ and accepting to pay his price $p_{j}$ :

1. those seeing Provider $j$ first, thus issuing a total demand (since they accept to pay $p_{j}$ ) $w\left(p_{j}\right) \lambda_{j} ;$

2. those seeing Provider $k \neq j$ (the competing provider) first, who refused to pay $p_{k}$ but would accept the price 
$p_{j}$ (possibly due to the acceptance threshold increase), forming a total demand level (smaller than $\lambda_{k}^{\text {ref }}$, and null when $\left.p_{k} \leq p_{j} / \alpha\right)$

$\lambda_{k}\left[w\left(p_{j} / \alpha\right)-w\left(p_{k}\right)\right]^{+}$,

where $x^{+}:=\max (0, x)$ for $x \in \mathbb{R}$;

3. and those seeing Provider $k$ first, who agreed to pay $p_{k}$ but were rejected because of Provider $k$ 's limited capacity, and who also agree to pay $p_{j}$, for a total demand

$\min \left(1, \frac{w\left(p_{j} / \alpha\right)}{w\left(p_{k}\right)}\right) \lambda_{k}^{\mathrm{sp}}$,

where $\lambda_{k}^{\text {sp }}$ is the part of the demand $w\left(p_{k}\right) \lambda_{k}$ that is spilled-over by Provider $k$.

The total demand $\lambda_{j}^{\mathrm{T}}\left(p_{j}, p_{k}\right)$ for Provider $j$ then equals the sum of the aforementioned components:

$$
\begin{aligned}
\lambda_{j}^{\mathrm{T}}\left(p_{j}, p_{k}\right):= & w\left(p_{j}\right) \lambda_{j}+\lambda_{k}\left[w\left(p_{j} / \alpha\right)-w\left(p_{k}\right)\right]^{+} \\
& +\min \left(1, \frac{w\left(p_{j} / \alpha\right)}{w\left(p_{k}\right)}\right) \lambda_{k}^{\mathrm{sp}}
\end{aligned}
$$

\subsection{Rejected users and uniqueness of flows}

When the total demand at an RSU exceeds its capacity, some requests are rejected: we assume that the RSU serves users up to its capacity, and that rejected requests are selected randomly among all arrived requests. Thus each request submitted to Provider $j$ has an identical probability of success $P_{j}$, that is simply given by

$P_{j}=\min \left(1, \frac{c}{\lambda_{j}^{T}}\right)$

so that the served traffic at RSU $j$ equals $\lambda_{j}^{\mathrm{T}} P_{j}=\min \left(c, \lambda_{j}^{\mathrm{T}}\right)$. Again, the probability $P_{j}$ depends on the price vector $\left(p_{i}, p_{k}\right)$. The corresponding revenue of provider $j$ is then

$R_{j}=p_{j} \min \left[c, \lambda_{j}^{\mathrm{T}}\left(p_{j}, p_{k}\right)\right]$.

The traffic $\lambda_{j}^{\mathrm{sp}}$, that is the part of $\lambda_{j}$ spilled over by Provider $j$ (and that will then enter the competitor's coverage area) also depends on both prices through the probability $P_{j}$, and equals

$\lambda_{j}^{\mathrm{sp}}=w\left(p_{j}\right) \lambda_{j}\left(1-P_{j}\right)$.

Regrouping all components of $\lambda_{j}^{T}$, the success probability equals

$P_{j}=\min \left(1, \frac{c}{w\left(p_{j}\right) \lambda_{j}+\left[w\left(p_{j} / \alpha\right)-w\left(p_{k}\right)\right]^{+} \lambda_{k}+\min \left[1, \frac{w\left(p_{j} / \alpha\right)}{w\left(p_{k}\right)}\right] \lambda_{k}^{\text {sp }}}\right)$.

If $p_{1}>p_{2} \alpha$ and $p_{1}>p_{2} / \alpha$, then those success probabilities should satisfy

$\left\{\begin{array}{l}P_{1}=\min \left(1, \frac{c}{w\left(p_{1}\right) \lambda_{1}+w\left(p_{1} / \alpha\right) \lambda_{2}-w\left(p_{1} / \alpha\right) \lambda_{2} P_{2}}\right) \\ P_{2}=\min \left(1, \frac{c}{w\left(p_{2}\right) \lambda_{2}+w\left(p_{2} / \alpha\right) \lambda_{1}-w\left(p_{1}\right) \lambda_{1} P_{1}}\right) .\end{array}\right.$
We obtain similar equations when $p_{1}<p_{2} / \alpha$ and $p_{1}<p_{2} \alpha$, by switching the roles of Providers 1 and 2 . Further, if $p_{2} / \alpha \leq$ $p_{1} \leq p_{2} \alpha$ then

$$
\left\{\begin{array}{l}
P_{1}=\min \left(1, \frac{c}{w\left(p_{1}\right) \lambda_{1}+w\left(p_{1} / \alpha\right) \lambda_{2}-w\left(p_{2}\right) \lambda_{2} P_{2}}\right) \\
P_{2}=\min \left(1, \frac{c}{w\left(p_{2}\right) \lambda_{2}+w\left(p_{2} / \alpha\right) \lambda_{1}-w\left(p_{1}\right) \lambda_{1} P_{1}}\right) .
\end{array}\right.
$$

Finally, if $p_{2} / \alpha \geq p_{1} \geq p_{2} \alpha$ (which can be the case for 350 $\alpha<1)$

$$
\left\{\begin{array}{l}
P_{1}=\min \left(1, \frac{c}{w\left(p_{1}\right) \lambda_{1}+w\left(p_{2}\right) \lambda_{2}-w\left(p_{2}\right) \lambda_{2} P_{2}}\right) \\
P_{2}=\min \left(1, \frac{c}{w\left(p_{2}\right) \lambda_{2}+w\left(p_{1}\right) \lambda_{1}-w\left(p_{1}\right) \lambda_{1} P_{1}}\right) .
\end{array}\right.
$$

Proposition 1. For any price vector $\left(p_{1}, p_{2}\right)$, the systems of 352 equations defined in (4), (5) and (6) have a unique solution. 353

Proof. See Appendix A.

\section{Single provider best response}

In this section, we study the situation when provider $k 356$ has fixed his price $p_{k}$, and provider $j$ wants to maximize his 357 revenue by setting appropriately his price $p_{j}$.

In our analysis, we will use the monotonicity of the demand function of a provider while its capacity remains unsaturated, which we establish now.

Lemma 1. The total demand $\lambda_{j}^{\mathrm{T}}$ of provider $j$ is a continuous function of his price $p_{j}$; that function is in addition nonincreasing while provider $j$ is not saturated (i.e., while $\lambda_{j}^{T}<c$ ).

Proof. See Appendix B.

For further analysis, we define the capacity saturation price of a provider as the price for which the total demand equals his capacity. Remark that this price depends on the price of his competitor.

Definition 1. The capacity saturation price of Provider $j$ is $p_{j}^{\mathrm{c}}\left(p_{k}\right):=\inf \left\{p \in\left[0, p_{\max }\right]: \lambda_{j}^{\mathrm{T}}\left(p, p_{k}\right)<c\right\}$.

Since $\lambda_{j}^{\mathrm{T}}\left(p_{\max }, p_{k}\right)=0$, for all $p_{k}$ we know that $p_{j}^{\mathrm{c}}\left(p_{k}\right)$ always exists. In addition we have $p_{j}^{c}\left(p_{k}\right)<p_{\max }$.

Lemma 1 implies that if $p_{j}^{\mathrm{c}}>0$, then $\lambda_{j}^{\mathrm{T}}\left(p_{j}^{\mathrm{c}}, p_{k}\right)=c$ and $p_{j} \leq p_{j}^{c} \Rightarrow \lambda_{j}^{\mathrm{T}} \geq c$.

When $\lambda_{j}^{\mathrm{T}}\left(0, p_{k}\right) \geq c, \lambda_{j}^{\mathrm{T}}\left(p_{j}^{\mathrm{c}}\right)=c$, hence $p_{j}^{\mathrm{c}}$ is the minimum price such that

$$
\left\{\begin{array}{l}
w\left(p_{j}^{\mathrm{c}}\right) \lambda_{j}+\lambda_{k}\left[w\left(p_{j}^{\mathrm{c}} / \alpha\right)-w\left(p_{k}\right)\right]^{+} \\
+\min \left(1, \frac{w\left(p_{j}^{\mathrm{c}} / \alpha\right)}{w\left(p_{k}\right)}\right) \lambda_{k}^{\mathrm{sp}}=c, \\
\lambda_{k}^{\mathrm{sp}}=w\left(p_{k}\right) \lambda_{k}\left[\frac{\left[w\left(p_{k} / \alpha\right)-w\left(p_{j}^{\mathrm{c}}\right)\right]^{+} \lambda_{j}+w\left(p_{k}\right) \lambda_{k}-c}{\left[w\left(p_{k} / \alpha\right)-w\left(p_{j}^{\mathrm{c}}\right)\right]^{+} \lambda_{j}+w\left(p_{k}\right) \lambda_{k}}\right]^{+} .
\end{array}\right.
$$

Solving this system then yields the capacity saturation price $p_{j}^{c}$. From Proposition 1 , the demand of Provider $j$ is

347 348 349 3 


\section{Fux et al./ Computer Networks $x x x$ (2015) $x x x-x x x$}

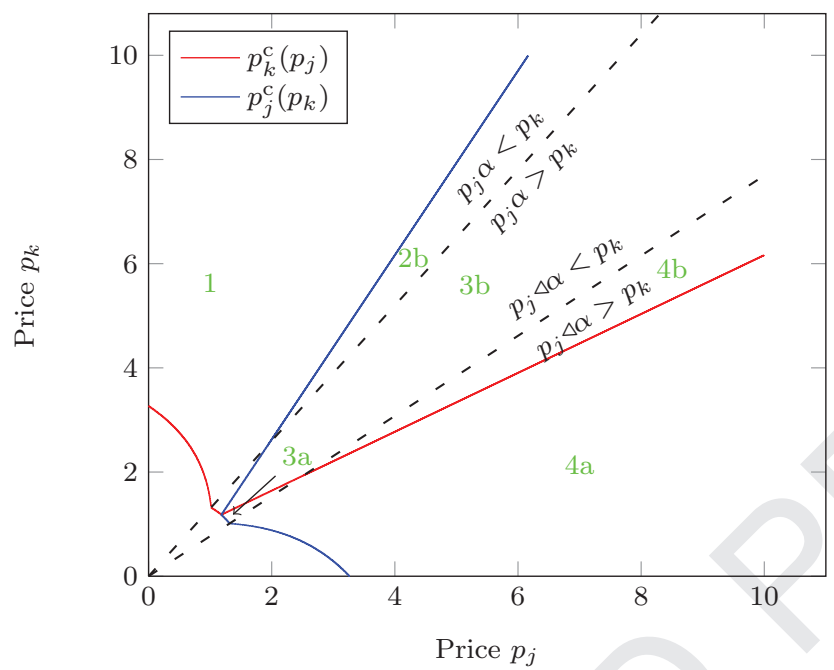

Fig. 3. Capacity saturation prices and the different price areas they form for $\alpha=1.3$ and $p_{k}=4$.

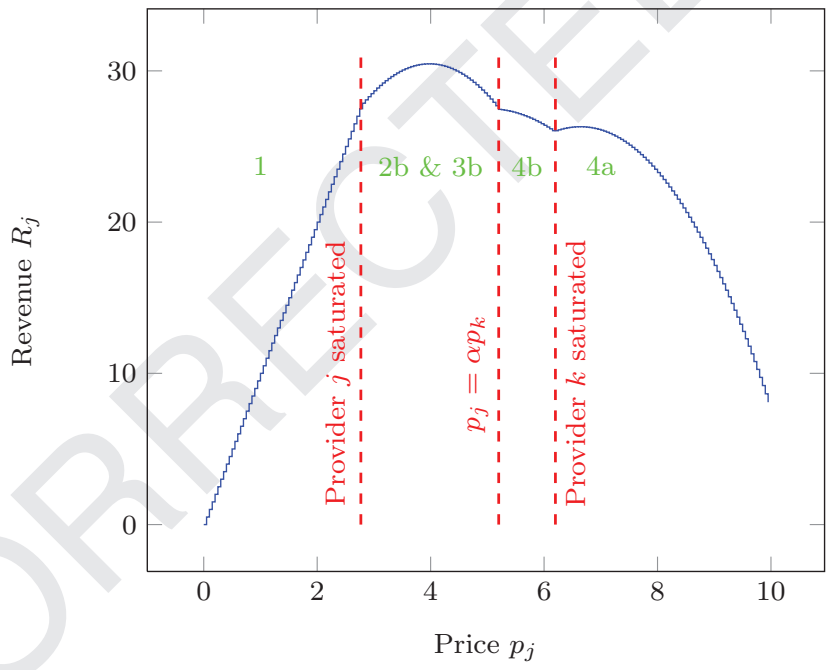

Fig. 4. Revenue of provider $j$ when $\alpha=1.3$ and $p_{k}=4$.

a continuous function of his price. Since we assumed that $\lambda_{j}^{\mathrm{T}}\left(0, p_{k}\right) \geq c$, and for $p_{j}=p_{\max }$ the demand equals zero, then the system (7) has a solution.

We now provide a piece-wise expression of the revenue function: the revenue function of each provider $j$ is continuous in his price (from the continuity of $\lambda_{j}^{\mathrm{T}}$ and of $P_{j}$ ), and can be expressed analytically on different segments.

1. When $\lambda_{j}^{\mathrm{T}}\left(p_{j}\right) \geq c\left(\right.$ or $p_{j} \leq p_{j}^{\mathrm{c}}\left(p_{k}\right)$ when $\left.p_{j}^{\mathrm{c}}\left(p_{k}\right)>0\right)$, the RSU capacity of provider $j$ is saturated, and thus his total load is simply

$\lambda_{j}^{T}=c$,

the revenue then equals

$R_{j}=p_{j} c$.

The corresponding segment of the revenue curve is the linear part as shown in Fig. 4, and corresponds in Fig. 3 to prices on the left of the capacity saturation curve of provider $j$.

2. If $p_{j}<p_{k} / \alpha$ and $p_{j}<p_{k} \alpha$, then provider $k$ cannot attract users having refused the price of provider $j$ :

$\lambda_{j}^{T}=w\left(p_{j}\right) \lambda_{j}+w\left(p_{j} / \alpha\right) \lambda_{k}-w\left(p_{k}\right) \lambda_{k}+\lambda_{k}^{s p}$,

with

$\lambda_{k}^{\mathrm{sp}}=\left[w\left(p_{k}\right) \lambda_{k}-c\right]^{+}$.

(a) If $p_{k}<p_{k}^{c}$, then the capacity of provider $k$ is saturated and $R_{j}=p_{j}\left(w\left(p_{j}\right) \lambda_{j}+w\left(p_{j} / \alpha\right) \lambda_{k}-c\right)$,

(b) Otherwise, provider $k$ is not saturated and $R_{j}=p_{j}\left(w\left(p_{j}\right) \lambda_{j}+w\left(p_{j} / \alpha\right) \lambda_{k}-w\left(p_{k}\right) \lambda_{k}\right)$.

Only case $2 \mathrm{~b}$ occurs on the example of Figs. 3 and 4.

3. If $p_{k} / \alpha \leq p_{j} \leq p_{k} \alpha$, then both providers are able to serve the refused traffic of each other:

$\lambda_{j}^{T}=w\left(p_{j}\right) \lambda_{j}+w\left(p_{j} / \alpha\right) \lambda_{k}-w\left(p_{k}\right) \lambda_{k}+\lambda_{k}^{s p}$, 
with

$\lambda_{k}^{\mathrm{sp}}=\left[w\left(p_{k}\right) \lambda_{k} \frac{w\left(p_{k}\right) \lambda_{k}+w\left(p_{k} / \alpha\right) \lambda_{j}-w\left(p_{j}\right) \lambda_{j}-c}{w\left(p_{k}\right) \lambda_{k}+w\left(p_{k} / \alpha\right) \lambda_{j}-w\left(p_{j}\right) \lambda_{j}}\right]^{+}$

(a) If $p_{k}<p_{k}^{c}$, then the capacity of provider $k$ is saturated and he gains

$$
\begin{aligned}
R_{j}= & p_{j}\left(w\left(p_{j}\right) \lambda_{j}+w\left(p_{j} / \alpha\right) \lambda_{k}\right. \\
& \left.-\frac{c}{w\left(p_{k}\right) \lambda_{k}+w\left(p_{k} / \alpha\right) \lambda_{j}-w\left(p_{j}\right) \lambda_{j}}\right),
\end{aligned}
$$

(b) Otherwise, provider $k$ is not saturated and his revenue is $R_{j}=p_{j}\left(w\left(p_{j}\right) \lambda_{j}+w\left(p_{j} / \alpha\right) \lambda_{k}-w\left(p_{k}\right) \lambda_{k}\right)$.

Figs. 3 and 4 illustrate both cases, with the only remark that in Fig. 4, cases 2b and 3b constitute one segment of the revenue curve (indeed, the expressions of the revenue function are identical in both cases).

4. If $p_{k} / \alpha \geq p_{j} \geq p_{k} \alpha$, then both providers do not serve the refused traffic:

$\lambda_{j}^{T}=w\left(p_{j}\right) \lambda_{j}+\lambda_{k}^{\mathrm{sp}}$,

with

$\lambda_{k}^{\mathrm{sp}}=\left[w\left(p_{k}\right) \lambda_{k}-c\right]^{+}$

(a) If $p_{k}<p_{k}^{c}$, then the capacity of provider $k$ is saturated and he gains

$R_{j}=p_{j}\left(w\left(p_{j}\right) \lambda_{j}+w\left(p_{k}\right) \lambda_{k}-c\right)$,

(b) Otherwise, provider $k$ is not saturated and the revenue is

$$
R_{j}=p_{j} w\left(p_{j}\right) \lambda_{j}
$$

5. If $p_{j}>p_{k} \alpha$ and $p_{j}>p_{k} / \alpha$, then the total load of provider $j$ is

$\lambda_{j}^{T}=w\left(p_{j}\right) \lambda_{j}+\frac{w\left(p_{j} / \alpha\right)}{w\left(p_{k}\right)} \lambda_{k}^{\mathrm{sp}}$,

where

$\lambda_{k}^{\mathrm{sp}}=\left[w\left(p_{k}\right) \lambda_{k} \frac{w\left(p_{k}\right) \lambda_{k}+w\left(p_{k} / \alpha\right) \lambda_{j}-w\left(p_{j}\right) \lambda_{j}-c}{w\left(p_{k}\right) \lambda_{k}+w\left(p_{k} / \alpha\right) \lambda_{j}-w\left(p_{j}\right) \lambda_{j}}\right]^{+}$.

(a) If $p_{k}<p_{k}^{c}$, then the capacity of provider $k$ is saturated and his revenue is

$$
\begin{aligned}
R_{j}= & p_{j}\left(w\left(p_{j}\right) \lambda_{j}+w\left(p_{j} / \alpha\right) \lambda_{k}\right. \\
& \left.\times \frac{w\left(p_{k}\right) \lambda_{k}+w\left(p_{k} / \alpha\right) \lambda_{j}-w\left(p_{j}\right) \lambda_{j}-c}{w\left(p_{k}\right) \lambda_{k}+w\left(p_{k} / \alpha\right) \lambda_{j}-w\left(p_{j}\right) \lambda_{j}}\right),
\end{aligned}
$$

(b) Otherwise, provider $k$ is not saturated and his revenue is simply

$$
R_{j}=p_{j} w\left(p_{j}\right) \lambda_{j}
$$

We can observe both cases in Figs. 3 and 4, where the plots are for a linear willingness-to-pay function $w(p)=[1-p / 10]^{+}, c=10$ and $\lambda_{1}=\lambda_{2}=11$. Unless stated otherwise, the same parameters are taken for all plots in the rest of the article.

Due to the complex form of the revenue function, computing the optimal price as a response to the price of the opponent leads to considering many subcases and hence appears analytically intractable. However, it is quite easy to compute it numerically on each segment and select the best one.

\section{Providers pricing game}

436

In this section we consider a non-cooperative game, where providers - the players - simultaneously choose their prices, trying to maximize their individual payoffs given by (2). Our aim is to find a Nash equilibrium (NE) of this game: a pair of prices $\left(\bar{p}_{1}, \bar{p}_{2}\right)$, such that no player can increase his payoff by unilaterally changing his price. The underlying assumption is that each provider knows in real time the current price of its competitor and is able to instantly adapt to it; but even if it is not the case, the providers can use the Nash equilibrium outcome as a prediction of their perfect information competition, and simultaneously charge equilibrium prices. Further, we investigate the situation where providers would decide to cooperate, trying to maximize the sum of their individual revenues (as a monopolist would do). We analyze how much the providers may lose in terms of total revenue by refusing to cooperate.

We first formally define the pricing game.

Definition 2. The providers pricing game is the 3-tuple

437 438 439 440 441 442 443 444 445 446 447 448 449 450 451 452 $G=(N, P, R)$,

where $N=\{1,2\}$ is the set of players (the two providers), $P=$ $\left(P_{1}, P_{2}\right)=\left(0, p_{\max }\right]^{2}$ is the space of players strategies and $R=$ $\left(R_{1}, R_{2}\right)$ is players payoffs or revenues given in (2).

We are interested in finding the Nash equilibrium of that pricing game.

Definition 3. A pair of prices $\left(\bar{p}_{1}, \bar{p}_{2}\right)$ is a Nash equilibrium for the pricing game if

$\left\{\begin{array}{l}R_{1}\left(\bar{p}_{1}, \bar{p}_{2}\right) \geq R_{1}\left(p_{1}, \bar{p}_{2}\right) \text { for all } p_{1} \in\left(0, p_{\max }\right], \\ R_{2}\left(\bar{p}_{1}, \bar{p}_{2}\right) \geq R_{2}\left(\bar{p}_{1}, p_{2}\right) \text { for all } p_{2} \in\left(0, p_{\max }\right] .\end{array}\right.$

Nash equilibria can be interpreted as predictions for the outcome of the competition between selfish entities, assumed rational and taking decisions simultaneously. For simplicity in this section we use the linear willingness-to-pay function, however the analogical results can be obtained for any other convex non-increasing function numerically.

\subsection{Large capacities regime}

In the remainder of this paper, we assume that RSU capacities exceed the total user flow (i.e., $c \geq \lambda_{j}+\lambda_{k}$ ). In particular, for any price profile RSU capacities are not saturated, and there is no spillover traffic.

This assumption is not necessarily restrictive; indeed in our previous study [11] we have established that at an equilibrium (if any) of the pricing game, no provider is saturated. Formally:

Proposition 2 ([11]). If $\left(\bar{p}_{j}, \bar{p}_{k}\right)$ is an equilibrium in the providers pricing game in the homogeneous flows case, then necessarily

$\left\{\begin{array}{l}\bar{p}_{j}>p_{j}^{c}\left(\bar{p}_{k}\right), \\ \bar{p}_{k}>p_{k}^{c}\left(\bar{p}_{j}\right) .\end{array}\right.$

For homogeneous user flows (i.e., $\lambda_{1}=\lambda_{2}$ ), we claim that if there is an equilibrium in the general capacities case, it is
469 470 471 472 473 474 475 476 
identical to the one with large capacities. Thus, the large capacity case contains all the equilibria we may have with arbitrary capacities; however those price profiles may not be equilibria in the general case.

\subsection{Providers competition}

The revenue expressions are again defined by segments (only two now, because of the large-capacity assumption):

$R_{j}= \begin{cases}p_{j}\left(w\left(p_{j}\right) \lambda_{j}+w\left(\frac{p_{j}}{\alpha}\right) \lambda_{k}-w\left(p_{k}\right) \lambda_{k}\right) & \text { if } p_{j} \leq p_{k} \alpha, \\ p_{j} w\left(p_{j}\right) \lambda_{j} & \text { otherwise. }\end{cases}$

In the rest of this section, we derive analytical expressions for the particular case of a linear willingness-to-pay function, of the form $w(p)=\left[1-p / p_{\max }\right]^{+}$for some constant $p_{\max }$.

We are interested in obtaining the best response function $\mathrm{BR}_{j}\left(p_{k}\right)$ of each provider $j$, that is the function indicating the optimal price to set as a response to the competitor's price $p_{k}$. For the best response function of provider $j$ we isolate only two candidate values from the revenue piecewise expressions above:

1. On the segment $\left[0, p_{k} \alpha\right]$, the best response of Provider $j$ is

$$
\mathrm{BR}_{j}^{a}=\min \left(p_{k} \alpha, \frac{p_{\max } \lambda_{j}+p_{k} \lambda_{k}}{2 \lambda_{j}+2 \lambda_{k} / \alpha}\right) .
$$

which is strictly below $p_{k} \alpha$ if $p_{k}>\frac{p_{\max } \lambda_{j}}{2 \lambda_{j} \alpha+\lambda_{k}}$.

2. On the segment $\left[p_{k} \alpha, \infty\right)$, Provider $j$ maximizes his revenue with

$\mathrm{BR}_{j}^{b}=\max \left(p_{k} \alpha, p_{\max } / 2\right)$,

which is strictly larger than $p_{k} \alpha$ if $p_{k}<\frac{p_{\max }}{2 \alpha}$.

Now remark that $\frac{p_{\max } \lambda_{j}}{2 \lambda_{j} \alpha+\lambda_{k}}<\frac{p_{\max }}{2 \alpha}$, hence because of the continuity of the revenue function:

- if $p_{k}<\frac{p_{\max } \lambda_{j}}{2 \lambda_{j} \alpha+\lambda_{k}}$ the best response is $\mathrm{BR}_{j}=p_{\max } / 2$;

- if $p_{k}>\frac{p_{\max }}{2 \alpha}$ the best response is $\mathrm{BR}_{j}=\frac{p_{\max } \lambda_{j}+p_{k} \lambda_{k}}{2 \lambda_{j}+2 \lambda_{k} / \alpha}$;

- for $\frac{p_{\max } \lambda_{j}}{2 \lambda_{j} \alpha+\lambda_{k}} \leq p_{k} \leq \frac{p_{\max }}{2 \alpha}$, we have to compare the two best-response candidates above, which we do now in the case of symmetric flows.

Proposition 3. Assume user flows are homogeneous, i.e., $\lambda_{1}=$ $\lambda_{2}=\lambda$, and consider a linear willingness-to-pay function $w(p)=\left[1-p / p_{\max }\right]^{+}$. Then the best-response of Provider $j$ is

$\mathrm{BR}_{j}= \begin{cases}\frac{p_{\max }+p_{k}}{2+2 / \alpha} & \text { if } p_{k} \geq p_{\max }\left(\sqrt{1+\frac{1}{\alpha}}-1\right) \\ \frac{p_{\max }}{2} & \text { otherwise. }\end{cases}$

Proof. Let us focus on the region where $\frac{p_{\max } \lambda_{j}}{2 \lambda_{j} \alpha+\lambda_{k}} \leq p_{k} \leq \frac{p_{\max }}{2 \alpha}$. In that region,

$R_{j}\left(\mathrm{BR}_{j}^{b}\right)=\frac{p_{\max }}{4} \lambda$

and

$$
\begin{aligned}
R_{j}\left(\mathrm{BR}_{j}^{a}\right) & =\frac{p_{\max }+p_{k}}{2+2 / \alpha} \lambda\left[1-\frac{1+\frac{p_{k}}{p_{\max }}}{2+2 / \alpha}-\frac{1+\frac{p_{k}}{p_{\max }}}{2 \alpha+2}+\frac{p_{k}}{p_{\max }}\right] \\
& =\frac{p_{\max }+p_{k}}{\alpha(2+2 / \alpha)^{2}} \lambda\left[\alpha+1+\alpha \frac{p_{k}}{p_{\max }}+\frac{p_{k}}{p_{\max }}\right] .
\end{aligned}
$$

The difference $R_{j}\left(\mathrm{BR}_{j}^{a}\right)-R_{j}\left(\mathrm{BR}_{j}^{b}\right)$ has the same sign as

$p_{k}^{2} \frac{1}{p_{\max }}+2 p_{k}-\frac{p_{\max }}{\alpha}$,

which is positive iff $p_{k} \geq p_{\max }\left(\sqrt{1+\frac{1}{\alpha}}-1\right)$. Finally we check that for all $\alpha$.

$1 /(2 \alpha+1)<\sqrt{1+\frac{1}{\alpha}}-1<1 /(2 \alpha)$,

which concludes the proof.

At a Nash equilibrium $\left(p_{1}^{*}, p_{2}^{*}\right)$, each provider is playing a best-response to the price set by the competitor. As a result, three types of equilibrium can occur:

- a symmetric Nash equilibrium, of the form $\left(\mathrm{BR}_{1}^{a}, \mathrm{BR}_{2}^{a}\right)$, leading to

$$
p_{1}^{*}=p_{2}^{*}=\frac{p_{\max }\left(2 \frac{\lambda_{j}^{2}}{\alpha}+\lambda_{k}^{2}+2 \lambda_{k} \lambda_{j}\right)}{4\left(\lambda_{k}+\frac{\lambda_{j}}{\alpha}\right)\left(\lambda_{j}+\frac{\lambda_{k}}{\alpha}\right)-\lambda_{j} \lambda_{k}} ;
$$

- a symmetric Nash equilibrium, of the form $\left(\mathrm{BR}_{1}^{b}, \mathrm{BR}_{2}^{b}\right)$, leading to

$p_{1}^{*}=p_{2}^{*}=\frac{p_{\max }}{2} ;$

- an asymmetric Nash equilibrium, with one provider (say, Provider $j$ ) playing $\mathrm{BR}_{j}^{a}$ and the other one playing $\mathrm{BR}_{k}^{b}$, leading to

$\left\{\begin{array}{l}p_{j}^{*}=\frac{p_{\max }\left(\lambda_{j}+\lambda_{k} / 2\right)}{2 \lambda_{j}+2 \lambda_{k} / \alpha} \\ p_{k}^{*}=p_{\max } / 2\end{array}\right.$

Considering again the homogeneous flow case, we determine the conditions on $\alpha$ for those price profiles to be Nash equilibria.

1. From Proposition 3, the symmetric equilibrium described in (8) exists only when

$p_{1}^{*} \geq p_{\max }\left(\sqrt{1+\frac{1}{\alpha}}-1\right)$,

i.e. when $\frac{2 / \alpha+3}{4(1+1 / \alpha)^{2}-1} \geq \sqrt{1+\frac{1}{\alpha}}-1$, which holds if and 536 only if $\alpha \geq \sqrt{\frac{4}{3}}$.

2. For the symmetric equilibrium described in (9), the condition of existence is:

$\left\{p_{\max } / 2 \leq p_{\max }\left(\sqrt{1+\frac{1}{\alpha}}-1\right)\right.$,

which is equivalent to $\alpha \leq 0.8$. 
Table 1

Nash equilibria of the pricing game, with homogeneous flows and a linear willingness-to-pay function.

\begin{tabular}{lll}
\hline Case & & Equilibrium prices \\
\hline$\alpha \leq 0.8$ & 1 equilibrium & $p_{1}^{*}=p_{2}^{*}=p_{\max } / 2$ \\
$\alpha \in[0.8, s]$ & 2 equilibria & $\left\{\begin{array}{l}p_{1}^{*}=3 p_{\max } /(4+4 / \alpha) \\
p_{2}^{*}=p_{\max } / 2\end{array}\right.$ \\
& & $\begin{array}{l}p_{1}^{*}=p_{\max } / 2 \\
p_{2}^{*}=3 p_{\max } /(4+4 / \alpha)\end{array}$ \\
& & \\
$\alpha \in\left(s, \sqrt{\frac{4}{3}}\right)$ & No equilibrium & \\
$\alpha \geq \sqrt{\frac{4}{3}}$ & 1 equilibrium & $p_{1}^{*}=p_{2}^{*}=p_{\max } \frac{2 / \alpha+3}{4(1+1 / \alpha)^{2}-1}$ \\
\hline
\end{tabular}

3. For the asymmetric equilibrium described in (10), the conditions of existence are:

$$
\left\{\begin{array}{l}
p_{\max } / 2 \geq p_{\max }\left(\sqrt{1+\frac{1}{\alpha}}-1\right), \\
\frac{3 p_{\max } / 2}{2+2 / \alpha} \leq p_{\max }\left(\sqrt{1+\frac{1}{\alpha}}-1\right) .
\end{array}\right.
$$

The first condition is equivalent to $\alpha \geq 0.8$, while the second one holds if and only if $\alpha \leq s$, where $s \approx 1.0766$.

Table 1 summarizes the equilibrium outcomes we can expect from the pricing game, depending on the value of $\alpha$. When $\alpha \leq 0.8$ both providers do not serve refused traffic and set prices as if there was no competitor. When $\alpha=1$, in the case of large capacities we have two similar equilibria, in which one provider charges a higher price than his competitor (and thus serves only users seeing him first) while the second provider serves traffic from both directions. When $\alpha$ increases, at those equilibria the low price increases: users who refused to pay the high price increase their willingnessto-pay before meeting the low-price provider, allowing the latter to make more revenue through a (moderate) price increase.
But at some $\alpha=s$, this lower equilibrium price becomes high enough to encourage the opponent to decrease his own price, in order to also serve some users who refused to pay the price of the opponent (those users become more valuable because of the large $\alpha$ ). This is the situation when the pricing game between providers has no equilibrium.

Finally, when $\alpha$ becomes high enough, each provider serves some users who refused the price of his competitor; the corresponding equilibrium is symmetric.

Two sets of best responses curves are shown in Fig. 5, for different $\alpha$ values illustrating the different types of equilibria. We observe that the prices in the symmetric equilibrium are lower than prices in asymmetric ones, which means that users accepting to pay more (through a larger $\alpha$ ) may lead to a situation where providers charge lower prices, a counterintuitive phenomenon. At the symmetric equilibrium, both providers serve some refused flows of each other due to the willingness-to-pay variation (when $\alpha>1$ ), while in asymmetric equilibria only one provider can serve the refused flow of its competitor; the former provider being then the one with the higher revenue. Note that the best response functions are discontinuous, implying that for some values of $\alpha$, there may be no Nash equilibrium.

The price decrease of the provider who had originally (for $\alpha=1$ ) the lowest price can be explained as follows: when the opponent decreases his price (that is lower at the symmetric equilibrium than at the original one) the refused flow reduces, and the influence of $\alpha$ is only on users from that flow who later accept to pay the proposed price. Thus, the provider is interested in lowering the price to attract more of those users.

Fig. 6 shows the corresponding equilibrium prices depending on $\alpha$ and Fig. 7 plots the equilibrium revenue of both providers. These figures confirm that for some values of $\alpha$, providers decrease their prices with respect to the reference case $\alpha=1$, resulting in a decrease of their total revenue. Surprisingly, for $\alpha$ approximately between 1.17 and 1.2, both providers set lower prices than when $\alpha=1$. When considering the average price per served used, the decrease (still

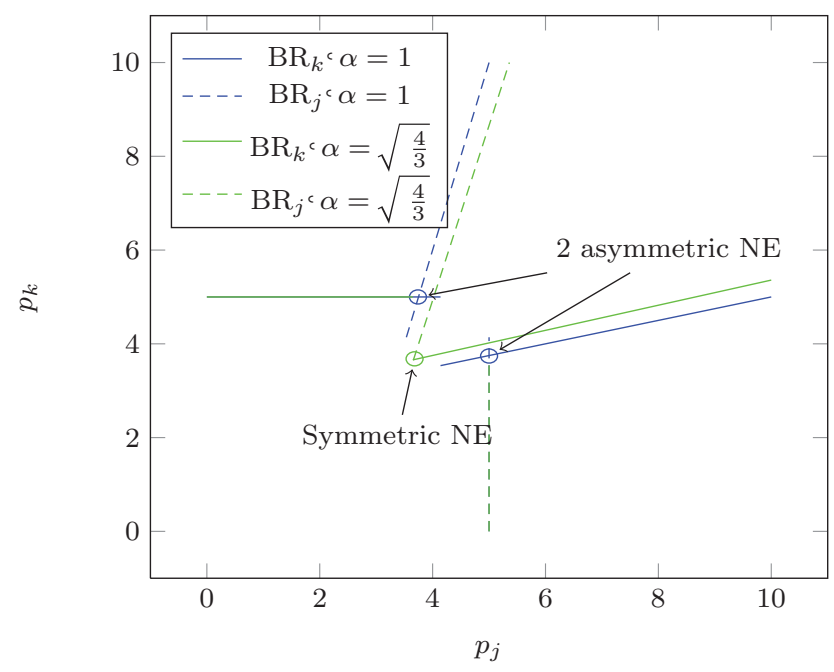

Fig. 5. Best responses curves for various $\alpha$. 


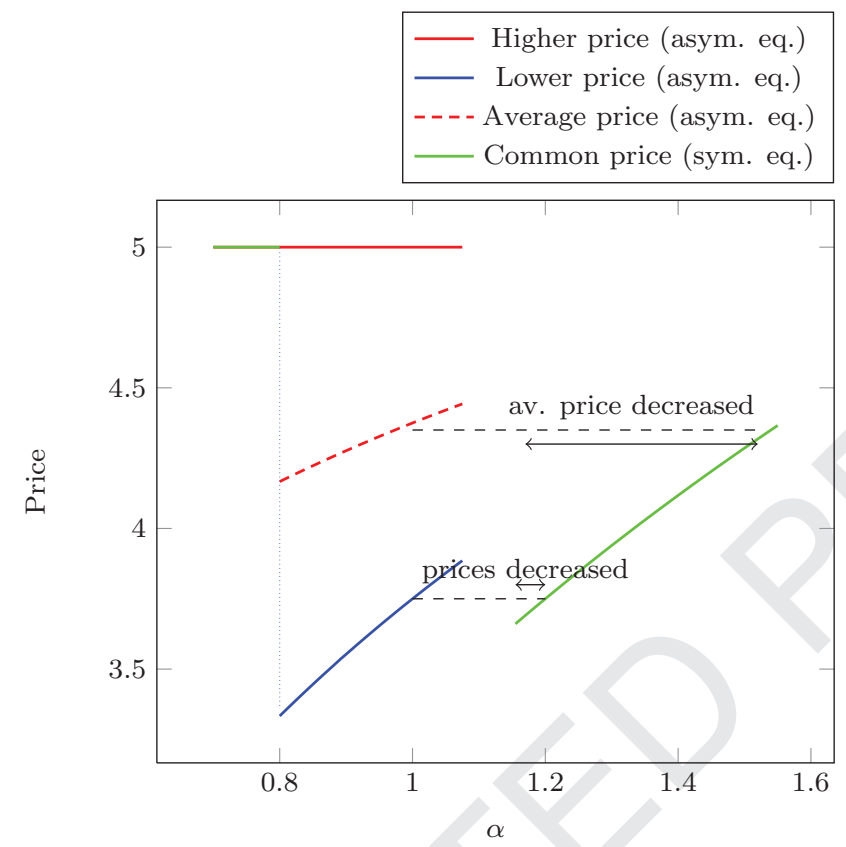

Fig. 6. Prices payed and their average values among all users at equilibrium. Note that for the symmetric equilibrium the average price is the (common) price charged by providers.

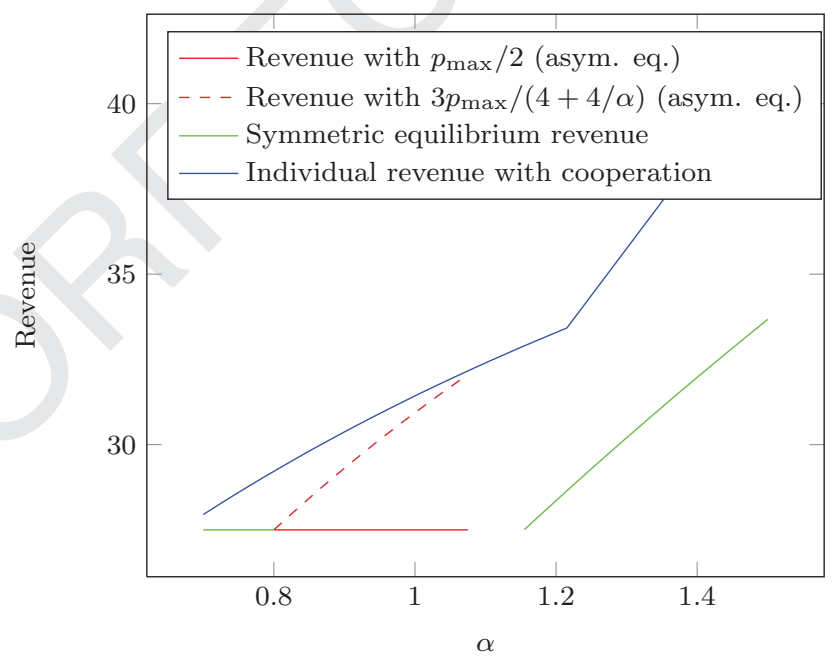

Fig. 7. Providers revenue in the cooperative and competitive equilibrium cases.

when compared to the case $\alpha=1$ ) occurs when $\alpha \in[1.17$, 1.52 ], approximately.

Now looking at the case $\alpha<1$, we notice that when $\alpha<$ 0.8 both providers charge the same price, which is the one they would have set had they been alone. This holds because for low $\alpha$, the users who refused the price of the first RSU they met would only accept very low prices for the second RSU, hence being of poor interest for the latter RSU owners. Providers are then better off focusing on their own direction flows.

Fig. 6 also illustrates that for approximately $1.075 \leq$ $\alpha \leq 1.17$, the game has no Nash equilibrium. This sit- uation arises when the refused flows at both sides become more important: due to the willingness-to-pay increase (when $\alpha>1$ ), users seeing the other provider first become a higher source of revenue and have more influence on each provider's pricing decision. For the evoked range of values for $\alpha$, this leads each provider to set a price below its competitor's until a point where focusing on one's flow-by setting large prices-is better, so that best-response curves do not intersect. Predicting the prices that are then chosen is difficult, since for any profile of prices at least one provider could do better by changing his price. 


\subsection{Cooperation among providers}

For comparison purposes we consider the situation where both providers cooperate when setting their prices, that is, the operators are no longer selfish, but rather have the common objective of maximizing the sum of their revenues. This implies that the operators share all the information about their pricing policies and act as a single entity.

We again assume homogeneous user flows, i.e., $\lambda_{1}=\lambda_{2}=$ $\lambda$. Without loss of generality we assume that the optimal prices are such that $p_{j} \leq p_{k}$.

To find such optimal prices, we again consider the two price zones where the revenue expressions differ:

1. First, if $p_{j} \leq \frac{p_{k}}{\alpha}$ and $p_{j} \leq p_{k} \alpha$, the total revenue is $R^{T}=p_{j}\left(w\left(p_{j}\right) \lambda+w\left(\frac{p_{j}}{\alpha}\right) \lambda-w\left(p_{k}\right) \lambda\right)+p_{k} w\left(p_{k}\right) \lambda$.

For a linear willingness-to-pay function, taking the partial derivatives yields

$$
\begin{aligned}
& \frac{\partial R^{T}}{\partial p_{j}}=\lambda\left(1-\frac{p_{j}}{p_{\max }}(2+2 / \alpha)+\frac{p_{k}}{p_{\max }}\right)=0, \\
& \frac{\partial R^{T}}{\partial p_{k}}=\lambda\left(1+\frac{p_{j}}{p_{\max }}-\frac{2 p_{k}}{p_{\max }}\right)=0,
\end{aligned}
$$

leading to the optimal price values

$$
\left\{\begin{array}{l}
\bar{p}_{j}=\frac{3 p_{\max }}{3+4 / \alpha} \\
\bar{p}_{k}=\frac{(3+2 / \alpha) p_{\max }}{3+4 / \alpha}
\end{array}\right.
$$

for $\alpha \leq 0.5+\sqrt{11 / 12}$. The corresponding total revenue is then

$$
{\overline{R^{\prime}}}^{T}=\frac{p_{\max } \lambda(9 \alpha+15+4 / \alpha)}{\alpha(3+4 / \alpha)^{2}} .
$$

2. If $\frac{p_{k}}{\alpha}<p_{j}\left(<p_{k} \alpha\right)$, the total revenue is:

$$
\begin{aligned}
R^{T}= & p_{j}\left(w\left(p_{j}\right) \lambda+w\left(\frac{p_{j}}{\alpha}\right) \lambda-w\left(p_{k}\right) \lambda\right) \\
& +p_{k}\left(w\left(p_{k}\right) \lambda+w\left(\frac{p_{k}}{\alpha}\right) \lambda-w\left(p_{j}\right) \lambda\right) .
\end{aligned}
$$

Again, partial derivatives give:

$$
\begin{aligned}
& \frac{\partial R^{T}}{\partial p_{j}}=\lambda\left(1-\frac{p_{j}}{p_{\max }}(2+2 / \alpha)+\frac{2 p_{k}}{p_{\max }}\right)=0, \\
& \frac{\partial R^{T}}{\partial p_{k}}=\lambda\left(1-\frac{p_{k}}{p_{\max }}(2+2 / \alpha)+\frac{2 p_{j}}{p_{\max }}\right)=0,
\end{aligned}
$$

and the optimal prices are

$$
\bar{p}_{j}=\bar{p}_{k}=\frac{p_{\max } \alpha}{2},
$$

yielding a total revenue

$$
{\overline{R^{\prime \prime}}}^{T}=\frac{p_{\max } \alpha \lambda}{2} \text {. }
$$

3. If $p_{k} \alpha<p_{j}<\frac{p_{k}}{\alpha}$, the total revenue is:

$$
R^{T}=p_{j} w\left(p_{j}\right) \lambda+p_{k} w\left(p_{k}\right) \lambda
$$

Again, partial derivatives give:

$$
\begin{aligned}
& \frac{\partial R^{T}}{\partial p_{j}}=\lambda\left(1-\frac{2 p_{j}}{p_{\max }}\right)=0, \\
& \frac{\partial R^{T}}{\partial p_{k}}=\lambda\left(1-\frac{2 p_{k}}{p_{\max }}\right)=0,
\end{aligned}
$$

and the optimal prices are

$\bar{p}_{j}=\bar{p}_{k}=\frac{p_{\max }}{2}$,

yielding a total revenue

$$
{\overline{R^{\prime \prime \prime}}}^{T}=\frac{p_{\max } \lambda}{4} \text {. }
$$

Now we derive the conditions to have ${\overline{R^{\prime}}}^{T} \geq{\overline{R^{\prime \prime}}}^{T}$ :

$$
\begin{aligned}
& \frac{p_{\max } \lambda(9 \alpha+15+4 / \alpha)}{\alpha(3+4 / \alpha)^{2}} \\
& \geq \frac{p_{\max } \alpha \lambda}{2} \Leftrightarrow 9 \alpha^{3}+6 \alpha^{2}-14 \alpha-8<0,
\end{aligned}
$$

and we have only one positive root $\bar{\alpha} \approx 1.215<0.5+$ $\sqrt{11 / 12}$. We have to compare ${\overline{R^{\prime \prime \prime}}}^{T}$ and ${\overline{R^{\prime}}}^{T}$. It appears that $\bar{R}^{\prime}{ }^{T}$ is always greater than $\overline{R^{\prime \prime \prime}}$ for positive $\alpha$ values.

Therefore,

$$
\begin{cases}\alpha \in[1, \bar{\alpha}] & R^{T}=\frac{p_{\max } \lambda(9 \alpha+15+4 / \alpha)}{\alpha(3+4 / \alpha)^{2}}, \\ \alpha>\bar{\alpha} & R^{T}=\frac{p_{\max } \alpha \lambda}{2} .\end{cases}
$$

Fig. 7 plots the individual revenues of both providers in the competition and cooperation cases assuming an equal share of cooperative revenue among providers for the latter, a reasonable assumption under homogeneous conditions (symmetric traffic flows, equal capacity, same willingnessto-pay function for users traveling in both directions). It appears that cooperation would improve the revenue of both providers, even the one that had the most favorable position in the asymmetric equilibrium.

\subsection{The impact on user surplus}

In this section we consider the equilibria of the pricing game from the point of view of users. Note that our model does not define a measure for individual customer efficiency: each customer is either fully served-getting a utility equal to his willingness-to-pay-or not served at all-getting zero utility; in case of congestion at an RSU, the unserved users are chosen uniformly among those accepting the proposed price. Thus, instead of efficiency we use user surplus, that is the difference between what users wanted to pay and what they actually payed. We focus here on the large capacity case. Recall that user willingness-to-pay varies in our scenario: we consider the initial willingness-to-pay as the reference: when $\alpha>1$, users served by the second provider met may actually pay more than they originally wanted to pay; in this case their surplus will be considered negative.

If we consider just one flow direction $\lambda_{j}$ and denote by $p_{j}$ the price of the first provider this flow meets, and by $p_{k}$ the price of the second one, then the positive part of users surplus is as follows:

$U S_{j}^{+}=\int_{p_{j}}^{p_{\max }} w(p) \lambda \mathrm{d} p+\int_{p_{k}}^{p_{j}}\left[w(p)-w\left(p_{j}\right)\right]^{+} \lambda \mathrm{d} p$, 


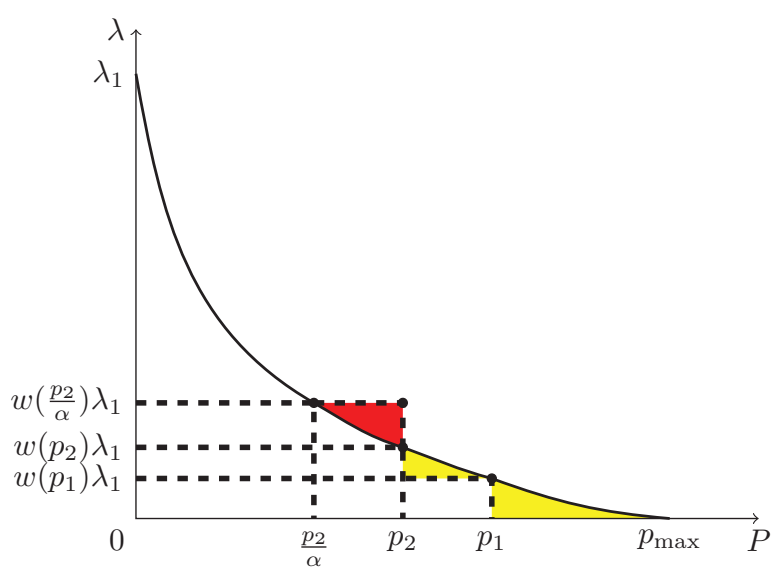

Fig. 8. Users surplus of $\lambda_{1}$ flow when $p_{1}>p_{2}$. (For interpretation of the references to colour in this figure, the reader is referred to the web version of this article).

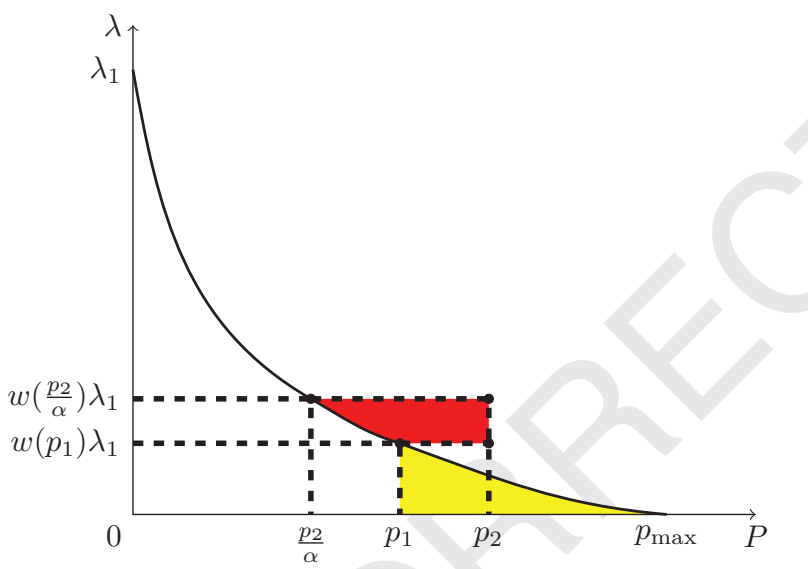

Fig. 9. Users surplus of $\lambda_{1}$ flow when $p_{2}>p_{1}$. (For interpretation of the references to colour in this figure, the reader is referred to the web version of this article). which includes surplus from users served by $j$, and by $k$. The negative part of users surplus is:

$$
\begin{aligned}
U S_{j}^{-}= & {\left[w\left(p_{k} / \alpha\right)-\max \left(w\left(p_{j}\right), w\left(p_{k}\right)\right)\right]^{+}\left(p_{k}-p_{k} / \alpha\right) \lambda } \\
& -\int_{p_{k} / \alpha}^{\min \left(p_{j}, p_{k}\right)}\left[w(p)-\max \left(w\left(p_{j}\right), w\left(p_{k}\right)\right)\right]^{+} \lambda \mathrm{d} p,
\end{aligned}
$$

which includes users refusing price $p_{j}$ and accepting a price $p_{k}$ higher than their original willingness-to-pay. Note that the expression of $U S_{j}^{-}$is general enough to cover both cases $p_{j}>$ $p_{k}$ and $p_{j}<p_{k}$.

Figs. 8 and 9 illustrate the logic behind the computation of user surplus when $p_{1}>p_{2}$ and $p_{1}<p_{2}$, respectively. The red surface is the negative part of user surplus (when they pay more than initially willing to), and yellow zones correspond to the positive part of users surplus.

With a linear willingness-to-pay function, we have

$U S_{j}^{+}=\left(p_{\max }-p_{j}\right) w\left(p_{j}\right) \frac{\lambda}{2}+\left(w\left(p_{k}\right)-w\left(p_{j}\right)\right)\left[p_{j}-p_{k}\right]^{+} \frac{\lambda}{2}$, and

$$
\begin{aligned}
U S_{j}^{-}= & \frac{\lambda}{2}\left(w\left(p_{k} / \alpha\right)-w\left(p_{k}\right)\right)\left(p_{k}-p_{k} / \alpha\right) \\
& -\frac{\lambda}{2}\left(w\left(p_{j}\right)-w\left(p_{k}\right)\right)\left[p_{k}-p_{j}\right]^{+}
\end{aligned}
$$

and the total user surplus is

$U S=U S_{j}^{+}+U S_{k}^{+}-U S_{j}^{-}-U S_{k}^{-}$.

Fig. 10 shows total users surplus for different $\alpha$ values for large capacities, in the similar settings as before. We can see that it is consistent with what we observed about the average price payed by user: for a whole range of $\alpha$ values, users surplus increases, which means that accepting to pay more led to the situation when (overall) users pay less.

\subsection{Numerical analysis for different willingness-to-pay functions}

Because of the complexity of the model, it is hard to prove analytically that for any function $w$ there is a range of $\alpha$ values such that a willingness-to-pay increases between the two providers met (by a factor $\alpha$ ) actually leads to a decrease in the prices set by providers. Note that it is possible to prove the existence of at least one symmetric equilibrium when $\alpha$ is large in the large-capacity case, but we cannot say anything about its quality.

In this section, we carry out a numerical analysis for some willingness-to-pay function examples, not restricting ourselves to linear ones. We are in particular interested in finding a minimum willingness-to-pay variation value $\bar{\alpha}$ for which a symmetric equilibrium appears, and compare the prices in this equilibrium with those for the case $\alpha=1$.

We consider the following functions:

- Linear: $w(p)=1-\frac{p}{p_{\max }}$

- Square: $w(p)=\left(1-\frac{p}{p_{\max }}\right)^{2}$

- Power Law $(C, n): w(p)=\frac{C}{C+p^{n}}$

- Exponential: $w(p)=\frac{1}{e^{p}}$

Table 2 shows provider prices at equilibrium, when there is no variation $(\alpha=1)$ and when the variation leads to a symmetric equilibrium. For the willingness-to-pay functions considered, which follow our convexity and monotonicity assumptions, we still observe a price decrease after some $\alpha$, illustrating that this phenomenon does not only occur with linear $w$ functions.

We also consider in Appendix $C$ the case where users moving in different directions modify their willingness-topay differently (i.e., one value of $\alpha$ for each direction). This scenario can correspond to situation when the highway stretch under consideration is close to a city area; users heading toward the city can anticipate to have several other connection opportunities (hence a low $\alpha$ ), while those leaving the city face a higher risk of not finding other (cheap) ways to connect (hence a higher $\alpha$ ).

\section{Discussion and perspectives}

This work studies competition between Internet access providers in vehicular networks in scenarios where users 


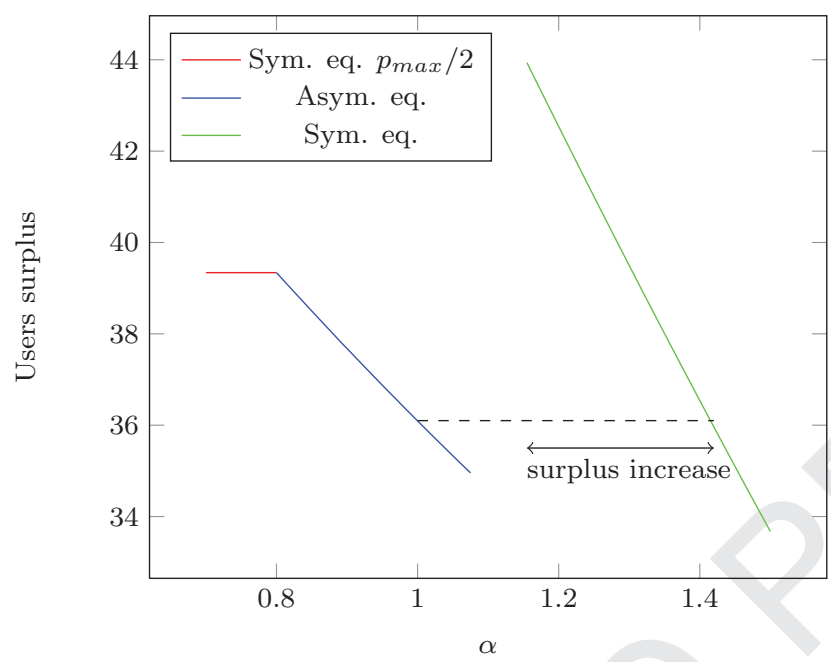

Fig. 10. Users surplus in equilibrium for various $\alpha$.

Table 2

Equilibrium prices decrease for different willingness-to-pay functions.

\begin{tabular}{llll}
\hline$w(p)$ & Equilibrium prices, $\alpha=1$ & Equilibrium prices, $\alpha=\bar{\alpha}$ & $\bar{\alpha}$ \\
\hline Linear & $(3.75,5.0)$ & $(3.68,3.68)$ & 1.16 \\
Square & $(2.35,3.33)$ & $(2.27,2.27)$ & 1.2 \\
Power law $(5,2.2)$ & $(1.35,1.92)$ & $(1.32,1.32)$ & 1.17 \\
Exponential & $(0.65,1.0)$ & $(0.59,0.59)$ & 1.25 \\
\hline
\end{tabular}

may change their pricing preferences as they travel, since they are less and less likely to be offered another connection possibility. We analyzed the optimal behavior of a provider, given the opponent's price fixed. This allowed us to characterize the outcomes (equilibria) of the competition among revenue-interested providers playing on prices.

Our finding is that the changes of users willingnessto-pay drastically impact the provider competition: users increasing their willingness-to-pay as they travel (a priori giving providers more latitude to make more revenues by increasing prices) can lead to counterintuitive situations where providers lower their prices and make fewer revenues, while reducing the average price payed by users. That phenomenon was observed for different types of willingness-to-pay functions.

The proposed modeling framework involves simplifying assumptions, which stems from the usual tension between having a realistic and insightful model and keeping it analytically tractable. First, we assume that all users undergo the same relative change in their price acceptance threshold (the price they accept to pay) between the two RSUs, i.e., the same $\alpha$. In a more detailed model, we may expect $\alpha$ to vary with the application involved, with the specific user ( $\alpha$ would then be modeled as a random variable), and/or with the initial price acceptance threshold value. Also, besides classical assumptions allowing to apply game theory (player rationality, perfect information about flow levels and opponent strategies), we assume that providers know users' willingness-topay and how it varies. Such an assumption can be justified as vehicular Internet providers may get to know the users' willingness-to-pay function through dynamic learning tech- niques and/or statistical inference. Then, a provider knowing the price of the opponent can estimate how the willingnessto-pay varies over time (the parameter $\alpha$ ): the fraction of users accepting to pay some price after refusing the price of the opponent indeed corresponds to a conditional probability that depends on both prices and on $\alpha$; the provider can thus vary his price and observe the demand level to estimate $\alpha$.

Despite the assumptions made, we believe that the proposed model provides insights on interesting phenomena, like the appearance of a symmetric equilibrium while there was not any when $\alpha$ equals 1 .

Natural follow ups for this work include:

- the analysis of larger network scenarios where each Internet provider owns a whole infrastructure of access points, spread (evenly or not) over the road, forming several connectivity islands; the analysis developed in this work for the case of 2-providers competition can be leveraged as a building block to address "larger" networks with higher number of providers and different network geometry. One possible approach could be to reduce such more complex scenarios to multiple 2-providers games. It is worth pointing out that including generic geometries for the deployment of RSUs may lead the competition outcomes to differ significantly, since relative position of providers' RSU have a drastic impact;

- the analysis of network scenarios where some a priori information is available on the providers' pricing strategies and/or the users become "strategic", that is, they become active players by properly setting their willingness-to-pay threshold (or entire function); this new setting, though, 
completely changes the structure of the competition and would call for a brand new modeling approach.

- the analysis of scenarios with consolidated incumbent providers and new providers willing to enter the market; this framework would call for changing the modeling approach resorting to leader-follower game representations.

- the analysis of network scenarios where the position of the RSUs is not pre-fixed, but rather each provider, besides setting the price for the service, may also decide where to deploy the network infrastructure. This setting requires ample modifications of the game theoretic framework.

\section{Acknowledgment}

This work has been partially funded by the Bretagne Region, through the ARED program, and by the Fondation Telecom through the "Futur\&Ruptures" program. We also would like to thank the anonymous reviewers for their valuable and helpful feedback.

\section{Appendix A. Proof of Proposition 1}

We first assume that $p_{1}>p_{2} \alpha$ and $p_{1}>p_{2} / \alpha$. Since the right-hand sides of the equations in (4) are continuous in $\left(P_{1}\right.$, $P_{2}$ ) and fall in the interval [0,1], Brouwer's fixed-point theorem [13] guarantees the existence of a solution to the system.

To establish uniqueness, remark that $P_{2}$ is uniquely defined by $P_{1}$ through the second equation in $(4)$, so $\left(P_{1}, P_{2}\right)$ is unique if $P_{1}$ is unique. But $P_{1}$ is a solution in $[0,1]$ of the fixed-point equation $x=g(x)$ with

$g(x):=\min \left(1, \frac{1}{a+b-b \min \left(1, \frac{1}{a+b+\epsilon-a x}\right)}\right)$,

where $\quad a=\frac{w\left(p_{1}\right) \lambda_{1}}{c}, \quad b=\frac{w\left(p_{1} / \alpha\right) \lambda_{2}}{c}, \quad$ and $\quad \epsilon=$ $\frac{\left(w\left(p_{2} / \alpha\right)-w\left(p_{1}\right)\right) \lambda_{1}+\left(w\left(p_{2}\right)-w\left(p_{1} / \alpha\right)\right) \lambda_{2}}{c}$ are all positive constants; we also assume $a>0$ and $b>0$ otherwise the problem is trivial. As a combination of two functions for the form $x \mapsto \min \left(1, \frac{1}{K_{1}-K_{2} x}\right), g$ is continuous, nondecreasing, strictly increasing only on an interval $[0, \bar{x}]$ (if any) - it is in addition convex on that interval -, and constant for $x \geq \bar{x}$ (note we can have $\bar{x}=0$ or $\bar{x} \geq 1$ ).

Assume $g(x)=x$ has a solution $\tilde{x} \in(0, \bar{x}]$. Then $g$ is leftdifferentiable at $\tilde{x}$, and

$g^{\prime}(\tilde{x})=\frac{\tilde{x}^{2} a b}{(a+b+\epsilon-a \tilde{x})^{2}} \leq \frac{\tilde{x}^{2} a}{(a+b+\epsilon-a \tilde{x})}$

where we used the fact that $\tilde{x} \leq 1$ (as a fixed point of $g$ ). Moreover, since $\tilde{x}$ is in the domain where $g$ is strictly increasing we have $\eta:=\frac{1}{a+b+\epsilon-a \tilde{x}} \leq 1$ on one hand, and $\tilde{x}=\frac{1}{a+b-b \eta}$ on the other side. Their combination yields $\tilde{x} \leq \frac{1}{a}$ and finally

$g^{\prime}(\tilde{x}) \leq \tilde{x} \leq 1$.

Remark also that $g^{\prime}(\tilde{x})<1$ if $\tilde{x}<1$. We finally use the fact that $g(0)>0$ to conclude that the curve $y=g(x)$ cannot meet the diagonal $y=x$ more than once: assume two intersection points $\tilde{x}_{1}<\tilde{x}_{2}$, then $g^{\prime}\left(\tilde{x}_{1}\right)<1$ thus the curves cross at $\tilde{x}_{1}$, another intersection point $\tilde{x}$ would imply $g^{\prime}\left(\tilde{x}_{2}\right)>1$ (recall $g$ is convex when strictly increasing), a contradiction. Hence the uniqueness of the fixed point and of the solution to (4).
By symmetry, we have the same kind of results when $p_{2} / \alpha \geq p_{1}$.

Then, we can also prove existence and uniqueness of a solution of system (5), when $p_{2} / \alpha \leq p_{1} \leq p_{2} \alpha$. Here we have

$g(x):=\min \left(1, \frac{1}{a+b-d \min \left(1, \frac{1}{d+a+\epsilon-a x}\right)}\right)$,

where $a=\frac{w\left(p_{1}\right) \lambda_{1}}{c}, \quad b=\frac{w\left(p_{1} / \alpha\right) \lambda_{2}}{c}, \quad d=\frac{w\left(p_{2}\right) \lambda_{2}}{c}$ and $\epsilon=$ $\frac{w\left(p_{2} / \alpha\right) \lambda_{1}-w\left(p_{1}\right) \lambda_{1}}{c}$ are all positive constants; we again assume $a>0$ and $b>0$ otherwise the problem is trivial.

Differentiating $g$ at $\tilde{x}$, we get

$g^{\prime}(\tilde{x})=\frac{\tilde{x}^{2} a d}{(a+d+\epsilon-a \tilde{x})^{2}} \leq \frac{\tilde{x}^{2} a}{(a+d+\epsilon-a \tilde{x})}$,

and the rest is similar to the case when $p_{1} / \alpha \geq p_{2}$.

Finally, we consider the case when $p_{2} / \alpha \geq p_{1} \geq p_{2} \alpha$. We have:

$g(x):=\min \left(1, \frac{1}{a+b-b \min \left(1, \frac{1}{b+a-a x}\right)}\right)$,

where $a=\frac{w\left(p_{1}\right) \lambda_{1}}{c}, b=\frac{w\left(p_{2}\right) \lambda_{2}}{c}$. The rest is similar to the first case.

\section{Appendix B. Proof of Lemma 1}

Recall that

$$
\begin{aligned}
\lambda_{j}^{\mathrm{T}}\left(p_{j}, p_{k}\right)= & w\left(p_{j}\right) \lambda_{j}+\lambda_{k}\left[w\left(p_{j} / \alpha\right)-w\left(p_{k}\right)\right]^{+} \\
& +\min \left(w\left(p_{k}\right), w\left(p_{j} / \alpha\right)\right) \lambda_{k}\left(1-P_{k}\right) .
\end{aligned}
$$

The components of the first line are trivially continuous and non-increasing in $p_{j}$ with our assumptions on $w(\cdot)$.

The continuity of $\lambda_{j}^{\mathrm{T}}\left(p_{j}, p_{k}\right)$ follows from the continuity of $P_{k}$ in the price vector $\left(p_{j}, p_{k}\right)$, established in the previous section.

To establish the monotonicity result, we distinguish four cases.

- If $p_{k}<p_{j} / \alpha$ and $p_{k}<p_{j} \alpha$, then we have

$$
\lambda_{j}^{\mathrm{T}}\left(p_{j}, p_{k}\right)=w\left(p_{j}\right) \lambda_{j}+w\left(p_{j} / \alpha\right) \lambda_{k}\left(1-P_{k}\right) .
$$

When $\lambda_{k}^{\mathrm{T}}<c$, then $P_{k}=1$ and $\lambda_{j}^{\mathrm{T}}$ is non-increasing in $p_{j}$.

Now if $\lambda_{k}^{\mathrm{T}}>c$ then from System (4) (this time with $k=2$, $j=1)$, we have $w\left(p_{k}\right) \lambda_{k}+w\left(p_{k} / \alpha\right) \lambda_{j}-w\left(p_{j}\right) \lambda_{j} P_{j}>c$ and

$$
\begin{aligned}
\lambda_{j}^{\mathrm{T}}\left(p_{j}, p_{k}\right)= & w\left(p_{j}\right) \lambda_{j}+w\left(p_{j} / \alpha\right) \lambda_{k}-w\left(p_{j} / \alpha\right) \\
& \times \lambda_{k} \frac{c}{w\left(p_{k}\right) \lambda_{k}+w\left(p_{k} / \alpha\right) \lambda_{j}-w\left(p_{j}\right) \lambda_{j} P_{j}} .
\end{aligned}
$$

Assuming that provider $j$ is not saturated, $P_{j}=1$. Then 5 18 858

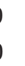
61 


$$
\begin{aligned}
& \lambda_{j}^{\prime T}\left(p_{j}, p_{k}\right)=w^{\prime}\left(p_{j}\right) \lambda_{j}+\frac{w^{\prime}\left(p_{j} / \alpha\right) \lambda_{k}}{\alpha}-\frac{w^{\prime}\left(p_{j} / \alpha\right) \lambda_{k}}{\alpha} \\
& -\frac{c}{w\left(p_{k}\right) \lambda_{k}+w\left(p_{k} / \alpha\right) \lambda_{j}-w\left(p_{j}\right) \lambda_{j}} \\
& \quad+w\left(p_{j} / \alpha\right) \lambda_{k} \frac{c w^{\prime}\left(p_{j}\right) \lambda_{j}}{\left(w\left(p_{k}\right) \lambda_{k}+w\left(p_{k} / \alpha\right) \lambda_{j}-w\left(p_{j}\right) \lambda_{j}\right)^{2}} \\
& \quad<w^{\prime}\left(p_{j}\right) \lambda_{j}+\frac{w^{\prime}\left(p_{j} / \alpha\right) \lambda_{k}}{\alpha}-\frac{w^{\prime}\left(p_{j} / \alpha\right) \lambda_{k}}{\alpha} \\
& \quad+w\left(p_{j} / \alpha\right) \lambda_{k} \frac{c w^{\prime}\left(p_{j}\right) \lambda_{j}}{\left(w\left(p_{k}\right) \lambda_{k}+w\left(p_{k} / \alpha\right) \lambda_{j}-w\left(p_{j}\right) \lambda_{j}\right)^{2}} \\
& \quad \leq 0,
\end{aligned}
$$

where the last inequality comes from the nonincreasingness of $w(\cdot)$.

- If $p_{j} / \alpha \leq p_{k} \leq p_{j} \alpha$ then

$$
\begin{aligned}
\lambda_{j}^{T}= & w\left(p_{j}\right) \lambda_{j}+w\left(p_{j} / \alpha\right) \lambda_{k} \\
& -\frac{c w\left(p_{k}\right) \lambda_{k}}{w\left(p_{k}\right) \lambda_{k}+w\left(p_{k} / \alpha\right) \lambda_{j}-w\left(p_{j}\right) \lambda_{j} P_{j}}
\end{aligned}
$$

Assuming that provider $j$ is not saturated and then $P_{j}=1$ we can differentiate in $p_{j}$ :

$$
\begin{aligned}
\frac{\mathrm{d} \lambda_{j}^{T}}{\mathrm{~d} p_{j}}= & w^{\prime}\left(p_{j}\right) \lambda_{j}+w^{\prime}\left(p_{j} / \alpha\right) \frac{\lambda_{k}}{\alpha} \\
& +\frac{c w\left(p_{k}\right) w^{\prime}\left(p_{j}\right) \lambda_{j} \lambda_{k}}{\left(w\left(p_{k}\right) \lambda_{k}+w\left(p_{k} / \alpha\right) \lambda_{j}-w\left(p_{j}\right) \lambda_{j}\right)^{2}} \leq 0,
\end{aligned}
$$

where $w^{\prime}$ is the derivative of $w$, and the last inequality comes from the fact that $w^{\prime}(\cdot) \leq 0$.

- If $p_{j} / \alpha \geq p_{k} \geq p_{j} \alpha$ (for $\alpha<1$ ) then

$$
\begin{aligned}
\lambda_{j}^{T}= & w\left(p_{j}\right) \lambda_{j}+w\left(p_{k}\right) \lambda_{k} \\
& -\frac{c w\left(p_{k}\right) \lambda_{k}}{w\left(p_{k}\right) \lambda_{k}+w\left(p_{j}\right) \lambda_{j}-w\left(p_{j}\right) \lambda_{j} P_{j}} .
\end{aligned}
$$

Assuming that provider $j$ is not saturated and then $P_{j}=$ 1:

$$
\frac{\mathrm{d} \lambda_{j}^{T}}{\mathrm{~d} p_{j}}=w^{\prime}\left(p_{j}\right) \lambda_{j} \leq 0 .
$$

- If $p_{k}>p_{j} \alpha$ and $p_{k}>p_{j} / \alpha$, we show that the success probability $P_{k}$ is non-decreasing in $p_{j}$ : applying System (4) (with $k=1, j=2$ ) we get that $P_{k}$ is the solution of the fixed-point equation $x=g(x)$, where the function $g$ can be written as

$$
g(x)=\min \left(1, \frac{c}{w\left(p_{k}\right) \lambda_{k}+w\left(p_{k} / \alpha\right) \lambda_{j}\left[1-\frac{c}{w\left(p_{j}\right) \lambda_{j}+w\left(p_{j} / \alpha\right) \lambda_{k}-w\left(p_{k}\right) \lambda_{k} x}\right]^{+}}\right)
$$

We then remark that, all else being equal, $g(x)$ is nondecreasing in $p_{j}$, so the solution $P_{k}$ of the fixed-point equation $g(x)=x$ is also non-decreasing in $p_{j}$.

As a result, when $p_{k} \geq p_{j} / \alpha$ the component $\min \left(w\left(p_{k}\right), w\left(p_{j} / \alpha\right)\right) \lambda_{k}\left(1-P_{k}\right)$ decreases with $p_{j}$, and so does $\lambda_{j}^{T}$.
Appendix C. Heterogeneous willingness-to-pay variations

899

In this section we assume that user pricing preferences change differently for both flow directions. Some users may for example move toward a city and thus expect to meet more APs, while the users moving in the opposite direction are risking not to meet any APs in the nearest future. The former may not increase much their willingness-to-pay, while the latter have higher risks to fail to establish Internet connection, and thus are more flexible in price perception.

Let us consider that the $\alpha$ values are different for two flows and that without loss of generality $\alpha_{1}$ value for users seeing Provider 1 first is bigger than for those, seeing first Provider 2, i.e., $\alpha_{1}=h \alpha_{2}=h \alpha$, for some $h \geq 1$.

Similarly to the case when $\alpha$ was common to both flow directions, we consider three cases:

1. If $p_{1}<\frac{p_{2}}{\alpha}$, then

$\left\{\begin{array}{l}R_{1}=p_{1}\left(w\left(p_{1}\right) \lambda_{1}+w\left(\frac{p_{1}}{\alpha h}\right) \lambda_{2}-w\left(p_{2}\right) \lambda_{2}\right), \\ R_{2}=p_{2} w\left(p_{2}\right) \lambda_{2}\end{array}\right.$

and for a linear $w(p)$

$\left\{\begin{array}{l}\mathrm{BR}_{1}^{a}=\frac{p_{\max } \lambda_{1}+p_{2} \lambda_{2}}{2 \lambda_{1}+\frac{2 \lambda_{2}}{\alpha h}}, \\ \mathrm{BR}_{2}^{b}=p_{\max } / 2 .\end{array}\right.$

and

$\mathrm{BR}_{1}^{a}\left(\mathrm{BR}_{2}^{b}\right)=\frac{p_{\max }\left(\lambda_{1}+1 / 2 \lambda_{2}\right)}{2 \lambda_{1}+\frac{2 \lambda_{2}}{\alpha h}}$.

This is valid for

$\alpha \leq \frac{\lambda_{1}+\sqrt{\lambda_{1}^{2}+4 \lambda_{2} / h\left(\lambda_{1}+1 / 2 \lambda_{2}\right)}}{2 \lambda_{1}+\lambda_{2}}$,

which in the homogeneous case is equivalent to

$\alpha \leq \frac{1+\sqrt{1+6 / h}}{3}$.

2. If $\frac{p_{2}}{\alpha} \leq p_{1} \leq p_{2} \alpha h$, then

$\left\{\begin{array}{l}R_{1}=p_{1}\left(w\left(p_{1}\right) \lambda_{1}+w\left(\frac{p_{1}}{\alpha h}\right) \lambda_{2}-w\left(p_{2}\right) \lambda_{2}\right), \\ R_{2}=p_{2}\left(w\left(p_{2}\right) \lambda_{2}+w\left(\frac{p_{2}}{\alpha}\right) \lambda_{1}-w\left(p_{1}\right) \lambda_{1}\right)\end{array}\right.$

and for a linear $w(p)$

$$
\left\{\begin{array}{l}
\mathrm{BR}_{1}^{a}=\frac{p_{\max } \lambda_{1}+p_{2} \lambda_{2}}{2 \lambda_{1}+\frac{2 \lambda_{2}}{\alpha h}}, \\
\mathrm{BR}_{2}^{a}=\frac{p_{\max } \lambda_{2}+p_{1} \lambda_{1}}{2 \lambda_{2}+\frac{2 \lambda_{1}}{\alpha}}, .
\end{array}\right.
$$

and

$$
\left\{\begin{array}{l}
\mathrm{BR}_{1}^{a}\left(\mathrm{BR}_{2}^{a}\right)=\frac{p_{\max }\left(2 \lambda_{1} \lambda_{2}+\frac{2 \lambda_{1}^{2}}{\alpha}+\lambda_{2}^{2}\right)}{\left(2 \lambda_{1}+\frac{2 \lambda_{2}}{\alpha h}\right)\left(2 \lambda_{2}+\frac{2 \lambda_{1}}{\alpha}\right)-\lambda_{1} \lambda_{2}}, \\
\mathrm{BR}_{2}^{a}\left(\mathrm{BR}_{1}^{a}\right)=\frac{p_{\max }\left(2 \lambda_{1} \lambda_{2}+\frac{2 \lambda_{2}^{2}}{\alpha h}+\lambda_{1}^{2}\right)}{\left(2 \lambda_{2}+\frac{2 \lambda_{1}}{\alpha}\right)\left(2 \lambda_{1}+\frac{2 \lambda_{2}}{\alpha h}\right)-\lambda_{1} \lambda_{2}} .
\end{array}\right.
$$

For this equilibrium the condition $\frac{p_{2}}{\alpha} \leq p_{1} \leq p_{2} \alpha h 922$ holds only if 


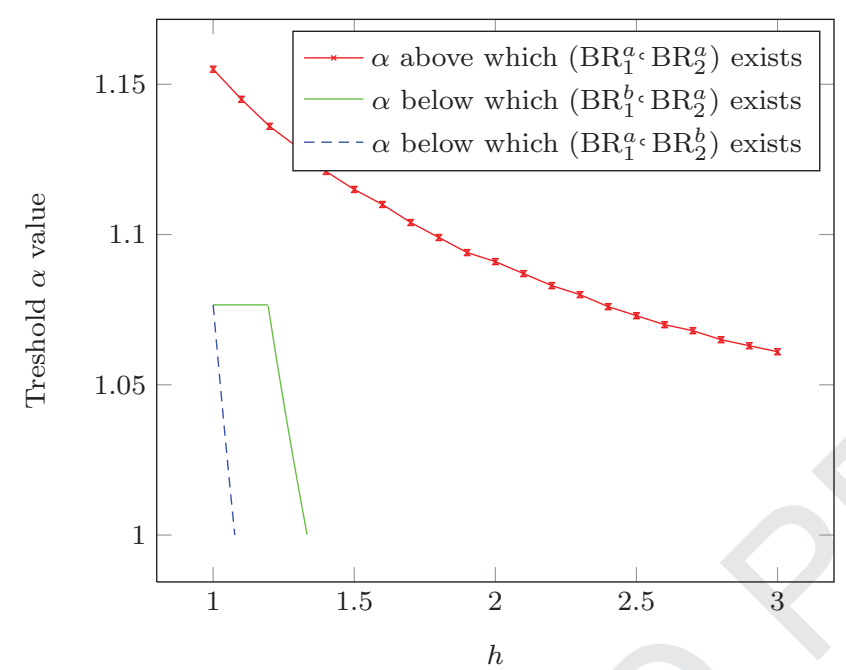

Fig. C.1. The different types of Nash equilibria in the pricing game when users seeing Provider 2 (resp. 1) first increase their price acceptance by a multiplicative $\alpha>1$ (resp. $h \alpha$ ) after seeing that provider.

$$
\left\{\begin{array}{l}
\alpha \geq \frac{-\lambda_{1}\left(\lambda_{1}-2 \lambda_{2}\right)+\sqrt{\lambda_{1}{ }^{2}\left(\lambda_{1}-2 \lambda_{2}\right)^{2}+8 \lambda_{2}{ }^{3} / h\left(\lambda_{2}+2 \lambda_{1}\right)}}{2 \lambda_{2}\left(\lambda_{2}+2 \lambda_{1}\right)}, \\
\alpha \geq \frac{-\lambda_{2}\left(\lambda_{2}-2 \lambda_{1}\right)+\sqrt{\lambda_{2}{ }^{2}\left(\lambda_{2}-2 \lambda_{1}\right)^{2}+8 \lambda_{1}{ }^{3} h\left(\lambda_{1}+2 \lambda_{2}\right)}}{2 h \lambda_{1}\left(\lambda_{1}+2 \lambda_{2}\right)},
\end{array}\right.
$$

What is different in this new scenario is that we have three types of equilibrium now $\left(\mathrm{BR}_{1}^{a}, \mathrm{BR}_{2}^{b}\right)$, and $\left(\mathrm{BR}_{1}^{a}, \mathrm{BR}_{2}^{b}\right)$ are not symmetric anymore. With homogeneous users flows we have the following conditions:

1. $\left(\mathrm{BR}_{1}^{a}, \mathrm{BR}_{2}^{b}\right)$ is an equilibrium when

$\alpha \geq \frac{1+\sqrt{1+24 / h}}{6}$.

3. If $p_{1}>p_{2} \alpha h$, then

$$
\left\{\begin{array}{l}
R_{1}=p_{1} w\left(p_{1}\right) \lambda_{1}, \\
R_{2}=p_{2}\left(w\left(p_{2}\right) \lambda_{2}+w\left(\frac{p_{2}}{\alpha}\right) \lambda_{1}-w\left(p_{1}\right) \lambda_{1}\right)
\end{array}\right.
$$

and for a linear $w(p)$

$$
\left\{\begin{array}{l}
\mathrm{BR}_{1}^{b}=p_{\max } / 2 \\
\mathrm{BR}_{2}^{a}=\frac{p_{\max } \lambda_{2}+p_{1} \lambda_{1}}{2 \lambda_{2}+\frac{2 \lambda_{1}}{\alpha}}, .
\end{array}\right.
$$

and

$$
\mathrm{BR}_{2}^{b}\left(\mathrm{BR}_{1}^{a}\right)=\frac{p_{\max }\left(\lambda_{2}+1 / 2 \lambda_{1}\right)}{2 \lambda_{2}+\frac{2 \lambda_{1}}{\alpha}},
$$

with the following condition on $\alpha$ to have $p_{1}>p_{2} \alpha h$ :

$$
\alpha<\frac{\lambda_{2}+\sqrt{\lambda_{2}^{2}}+4 \lambda_{1} h\left(\lambda_{2}+1 / 2 \lambda_{1}\right)}{2^{\sim} h\left(\lambda_{2}+1 / 2 \lambda_{1}\right)},
$$

or in the homogeneous flows case

$$
\alpha<\frac{1+\sqrt{1+6 h}}{3 h} .
$$

$$
\left\{\begin{array}{l}
\mathrm{BR}_{1}^{a}\left(\mathrm{BR}_{2}^{b}\right)<p_{\max }\left(\sqrt{1+\frac{1}{\alpha h}}-1\right), \\
\mathrm{BR}_{2}^{b}\left(\mathrm{BR}_{1}^{a}\right) \geq p_{\max }\left(\sqrt{1+\frac{1}{\alpha}}-1\right), \\
\alpha<\frac{1+\sqrt{1+6 / h}}{3} .
\end{array}\right.
$$

or $\alpha<\min \left\{s / h, \frac{1+\sqrt{1+6 / h}}{3}\right\}$.

$$
\left\{\begin{array}{l}
\mathrm{BR}_{1}^{a}\left(\mathrm{BR}_{2}^{a}\right) \geq p_{\max }\left(\sqrt{1+\frac{1}{\alpha h}}-1\right), \\
\mathrm{BR}_{2}^{a}\left(\mathrm{BR}_{1}^{a}\right) \geq p_{\max }\left(\sqrt{1+\frac{1}{\alpha}}-1\right), \\
\alpha \geq \frac{1+\sqrt{1+24 / h}}{6}
\end{array}\right.
$$

This set of inequalities is not solvable for $\alpha h$, but for each specific value of $h$ we can find numerically a condition on $\alpha$ for the conditions to hold. This dependence is presented on Fig. C.1 
3. $\left(\mathrm{BR}_{1}^{b}, \mathrm{BR}_{2}^{a}\right)$ is an equilibrium when

$$
\left\{\begin{array}{l}
\operatorname{BR}_{1}^{b}\left(\mathrm{BR}_{2}^{a}\right) \geq p_{\max }\left(\sqrt{1+\frac{1}{\alpha h}}-1\right), \\
\mathrm{BR}_{2}^{a}\left(\mathrm{BR}_{1}^{b}\right)<p_{\max }\left(\sqrt{1+\frac{1}{\alpha}}-1\right), \\
\alpha<\frac{1+\sqrt{1+6 h}}{3 h},
\end{array}\right.
$$

or $\alpha<\min \left\{s, \frac{1+\sqrt{1+6 h}}{3 h}\right\}$.

Fig. C.1 shows threshold $\alpha$ values for different $h$, showing whether there exists a particular type of equilibrium. The figure suggests that there is no pair of $\alpha$ and $h$ such that all three types of equilibria exist.

\section{References}

[1] E. Altman, A. Kumar, C. Singh, R. Sundaresan, Spatial SINR games combining base station placement and mobile association, in: Proceedings of IEEE INFOCOM, 2009, doi:10.1109/INFCOM.2009.5062081.

[2] J. Barrachina, P. Garrido, M. Fogue, F. Martinez, J.-C. Cano, C. Calafate, P. Manzoni, Road side unit deployment: a densitybased approach, IEEE Intell. Transp. Syst. Mag. 5 (3) (2013) 30-39, doi:10.1109/MITS.2013.2253159.

[3] F. Borgonovo, A. Capone, M. Cesana, L. Fratta, ADHOC MAC: new MAC architecture for ad-hoc networks providing efficient and reliable pointto-point and broadcast services, Wirel. Netw. 10 (4) (2004) 359-366, doi:10.1023/B:WINE.0000028540.96160.8a.

[4] E.S. Cavalcante, A.L.L. Aquino, G.L. Pappa, A.A.F. Loureiro, Roadside unit deployment for information dissemination in a VANET: an evolutionary approach, in: Proceedings of GECCO Companion, 2012.

[5] D.E. Charilas, A.D. Panagopoulos, A survey on game theory applications in wireless networks, Comput. Netw. 54 (18) (2010) 3421-3430.

[6] L.A. DaSilva, Pricing for QoS-enabled networks: a survey, IEEE Commun. Surv. Tutor. 3 (2) (2000) 2-8.

[7] J. Eriksson, H. Balakrishnan, S. Madden, Cabernet: vehicular content delivery using WiFi, in: Proceedings of ACM MobiCom, New York, NY, USA, 2008, pp. 199-210, doi:10.1145/1409944.1409968.

[8] I. Filippini, F. Malandrino, G. Dan, M. Cesana, C. Casetti, I. Marsh, Noncooperative RSU deployment in vehicular networks, in: Proceedings of Wireless On-demand Network Systems and Services,WONS, 2012.

[9] V. Fux, P. Maillé, Incentivizing efficient load repartition in heterogeneous wireless networks with selfish delay-sensitive users, in: Proceedings of Internet Charging and QoS Technologies,ICQT, 2013.

[10] V. Fux, P. Maillé, J.-M. Bonnin, N. Kaci, Efficiency or fairness: managing applications with different delay sensitivities in heterogeneous wireless networks, in: Proceedings of IEEE World of Wireless Mobile and Multimedia Networks, WoWMoM, 2013.

[11] V. Fux, P. Maillé, M. Cesana, Price competition between road side units operators in vehicular networks, in: Proceedings of IFIP Networking, Trondheim, Norway, 2014. Version with appendices available online: https://hal.archives-ouvertes.fr/hal-00967110

[12] H. Hartenstein, K.P. Laberteaux, A tutorial survey on vehicular ad-hoc networks, IEEE Commun. Mag. 46 (6) (2008) 164-171, doi:10.1109/MCOM.2008.4539481.

[13] S. Kakutani, A generalization of Brouwer's fixed point theorem, Duke Math. J. 8 (1941) 457-459.

[14] U. Lee, E. Magistretti, M. Gerla, P. Bellavista, A. Corradi, Dissemination and harvesting of urban data using vehicular sensing platforms, IEEE Trans. Veh. Technol. 58 (2) (2009) 882-901, doi:10.1109/TVT.2008.928899.

[15] P. Maillé, B. Tuffin, Competition among providers in loss networks, Annals. Oper. Res. 199 (2012).

[16] P. Maillé, B. Tuffin, Telecommunication Network Economics: From Theory to Applications, Cambridge University Press, 2014.

[17] D. Niyato, E. Hossain, Competitive pricing in heterogeneous wireless access networks: issues and approaches, IEEE Netw. 22 (6) (2008).
[18] D. Niyato, E. Hossain, A unified framework for optimal wireless access for data streaming over vehicle-to-roadside communications, IEEE Trans. Veh. Technol. 59 (6) (2010) 3025-3035, doi:10.1109/TVT.2010.2048769.

[19] D. Niyato, E. Hossain, M. Hassan, Game-theoretic models for vehicular networks, in: Y. Zhang, M. Guizani (Eds.), Game Theory for Wireless Communications and Networking, CRC Press, 2011, pp. 61-98, doi:10.1201/b10975-6.

[20] D. Niyato, E. Hossain, Ping Wang, Competitive wireless access for data streaming over vehicle-to-roadside communications, in: Proceedings of IEEE GLOBECOM, 2009, pp. 1-6, doi:10.1109/GLOCOM.2009.5425820.

[21] J. Ott, D. Kutscher, The "drive-thru" architecture: WLAN-based internet access on the road, in: Proceedings of IEEE Vehicular Technology Conference,VTC, 5, 2004.

[22] J. Ott, D. Kutscher, Drive-thru internet: IEEE $802.11 \mathrm{~b}$ for "automobile" users, in: Proceedings of IEEE INFOCOM, 2004.

[23] J. Ott, D. Kutscher, A disconnection-tolerant transport for drive-thru internet environments, in: Proceedings of IEEE INFOCOM, 2005.

[24] J. Ott, D. Kutscher, Exploiting regular hot-spots for drive-thru internet, in: Paul Mller, Reinhard Gotzhein, Jens B. Schmitt (Eds.), KiVS, Informatik Aktuell, Springer, 2005, pp. 218-229.

[25] J. Ott, D. Kutscher, A modular access gateway for managing intermittent connectivity in vehicular communications., Eur. Trans. Telecommun. 17 (2) (2006) 159-174.

[26] J. Ott, D. Kutscher, M. Koch, Towards automated authentication for mobile users in WLAN hot-spots, in: In Proceedings of Vehicular Technology Conference, VTC Fall, 2005.

[27] W. Saad, Zhu Han, A. Hjorungnes, D. Niyato, E. Hossain, Coalition formation games for distributed cooperation among roadside units in vehicular networks, IEEE JSAC 29 (1) (2011) 48-60, doi:10.1109/JSAC.2011.110106.

[28] T. Small, Z.J. Haas, The shared wireless infostation model: a new ad-hoc networking paradigm (or where there is a whale, there is a way), in: Proceedings of Annual International Conference on Mobile Computing and Networking, ACM MobiHoc, 2003, pp. 233-244, doi: $10.1145 / 778415.778443$. New York, NY, USA

[29] R. Trestian, O. Ormond, G.-M. Muntean, Game theory-based network selection: solutions and challenges, IEEE Commun. Surv. Tutor. (2012).

[30] O. Trullols, M. Fiore, C. Casetti, C.F. Chiasserini, J.M.Barcelo Ordinas, Planning roadside infrastructure for information dissemination in intelligent transportation systems, Comput. Commun. 33 (4)(2010) 432442. http://dx.doi.org/10.1016/j.comcom.2009.11.021.

[31] B. Tuffin, Charging the internet without bandwidth reservation: an overview and bibliography of mathematical approaches, J. Inf. Sci. Eng. 19 (2003) 765-786.

[32] T. Yan, W. Zhang, G. Wang, Y. Zhang, Access points planning in urban area for data dissemination to drivers, IEEE Trans. Veh. Technol. (2013), doi:10.1109/TVT.2013.2272724.

[33] K. Yang, S. Ou, H.-H. Chen, J. He, A multihop peer-communication protocol with fairness guarantee for IEEE 802.16-based vehicular networks, IEEE Trans. Veh. Technol. 56 (6) (2007) 3358-3370, doi:10.1109/TVT.2007.906875.

[34] X. Yang, J. Liu, N.F. Vaidya, F. Zhao, A vehicle-to-vehicle communication protocol for cooperative collision warning, in: Proceedings of Mobile and Ubiquitous Systems, MOBIQUITOUS, 2004, pp. 114-123, doi:10.1109/MOBIQ.2004.1331717.

[35] Y. Zhang, J. Zhao, G. Cao, On scheduling vehicle-roadside data access, in: Proceedings of ACM VANET, ACM, New York, NY, USA, 2007, pp. 9-18, doi:10.1145/1287748.1287751.

[36] Z. Zheng, Z. Lu, P. Sinha, S. Kumar, Ensuring predictable contact opportunity for scalable vehicular internet access on the go, IEEE/ACM Trans. Netw. (2015), doi:10.1109/TNET.2014.2309991. In Press.

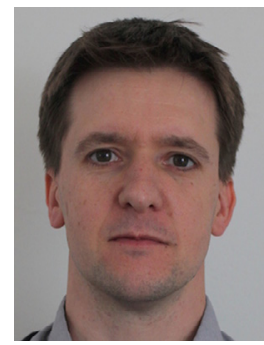

Patrick Maillé graduated from Ecole polytechnique and Telecom ParisTech, France. He has been with the Network, Security, Multimedia department of Telecom Bretagne since 2002, where he obtained his Ph.D. in applied mathematics in 2005. His research interests are in all economic aspects of telecommunication networks, from pricing schemes at the user level, to auctions for spectrum and regulatory issues (net neutrality, search neutrality). He recently co-authored the book "Telecommunication Network Economics", published by Cambridge University Press. 


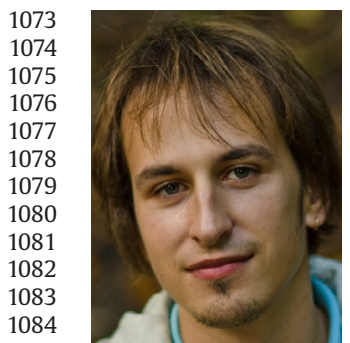

Vladimir Fux graduated in 2011 in Applied Mathematics and Computer Science from the SaintPetersburg State University, Russia, and worked on sampling algorithms for web graphs as an intern at Inria, France. Since 2011 he has been a Ph.D. student in the Network, Security and Multimedia department of Telecom Bretagne, France, investigating game-theory approaches to study and influence interactions among self-interested agents in wireless networks. His research interest include game theory, economics, wireless networks and information retrieval.

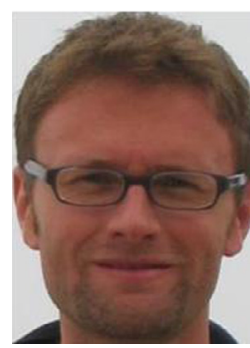

Matteo Cesana is currently an Assistant Professor with the Dipartimento di Elettronica, Informazione e Bioingegneria of the Politecnico di Milano, Italy. He received his MS degree in Telecommunications Engineering and his Ph.D. degree in Information Engineering from Politecnico di Milano in July 2000 and in September 2004, respectively. From September 2002 to March 2003 he was a visiting researcher at the Computer Science Department of the University of California in Los Angeles (UCLA). His research activities are in the field of design, optimization and performance evaluation of wireless networks with a specific focus on wireless sensor networks and cognitive radio networks. Dr. Cesana is an Associate Editor of the Ad Hoc Networks Journal (Elsevier).
1085

1086

1087

1088

1089

1090

1091

1092

1093

1094

1095

1096

1097

1098

1099

1100 